\title{
Coronagraphic search for wide substellar companions among members of the Ursa Major moving group $\star, \star \star$
}

\author{
M. Ammler-von Eiff ${ }^{1,2,3,4,5,6}$, A. Bedalov ${ }^{3,7}$, C. Kranhold ${ }^{2}$, M. Mugrauer ${ }^{3}$, T. O. B. Schmidt ${ }^{3,8}$, \\ R. Neuhäuser ${ }^{3}$, and R. Errmann ${ }^{3,9}$

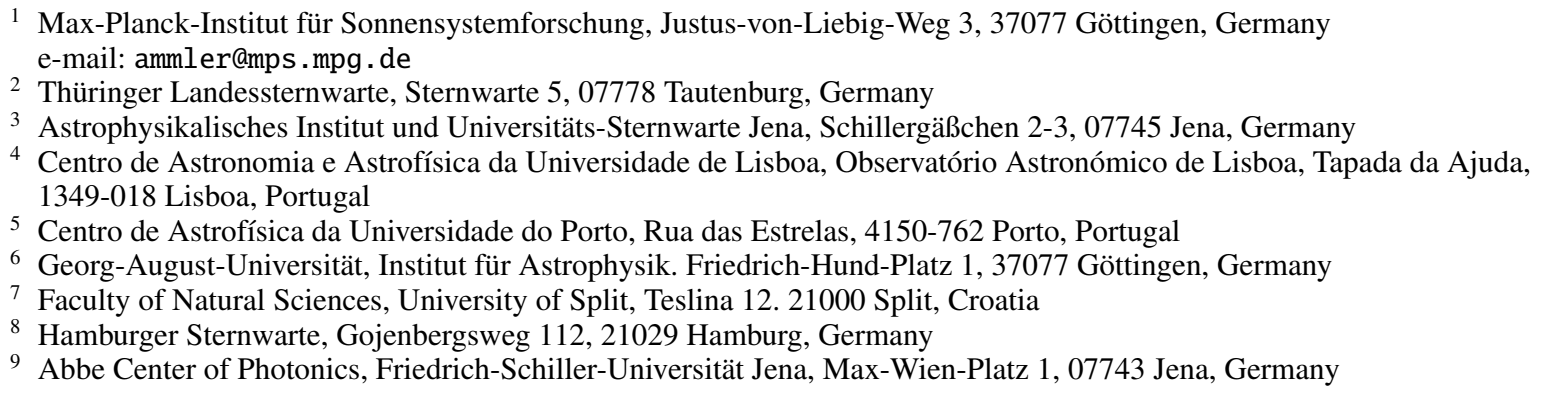

Received 28 May 2015 / Accepted 9 March 2016

\begin{abstract}
Context. We present the results of a survey to detect low-mass companions of Ursa Major (UMa) group members, carried out in 2003-2006 with NACO at the ESO VLT. While many extra-solar planets and planetary candidates have been found in close orbits around stars by the radial velocity and the transit methods, direct detections at wider orbits are rare. The UMa group, a young nearby stellar association at an age of about $200-600 \mathrm{Myr}$, has not yet been addressed as a whole although its members represent a very interesting sample to search for and characterize substellar companions by direct imaging.

Aims. Our goal was to find or to provide detection limits on wide substellar companions around nearby UMa group members using high-resolution imaging.

Methods. We searched for faint companions around 20 UMa group members within $30 \mathrm{pc}$. The primaries were placed below a semitransparent coronagraph, a rarely used mode of NACO, to increase the dynamic range of the images. In most cases, second epoch images of companion candidates were taken to check whether they share common proper motion with the primary.

Results. Our coronagraphic images rule out substellar companions around the stars of the sample. A typical dynamical range of $13-15 \mathrm{mag}$ in the $K_{\mathrm{s}}$ band was achieved at separations beyond $3^{\prime \prime}$ from the star. Candidates as faint as $K_{\mathrm{s}} \approx 20$ were securely identified and measured. The survey is most sensitive between separations of 100 and 200 au but only on average because of the very different target distance. Field coverage reaches about 650 au for the most distant targets. Most of the 200 candidates detected in the covered fields are visible in two epochs and were rejected because they are distant background objects.
\end{abstract}

Key words. binaries: visual - stars: imaging - brown dwarfs - open clusters and associations: individual: Ursa Major solar neighborhood

\section{Introduction}

To date almost 1,300 brown dwarfs have been found, classified with spectral types L, T, and Y (Kirkpatrick 2008; Cushing et al. $2011)^{1}$. This number has been surpassed by indirect detections in the planetary regime - more than 600 by radial velocity variations and more than 1200 by the transit method ${ }^{2}$.

While indirect methods are most sensitive to objects in very close orbits around the targets, direct imaging detects objects in wider orbits and thus is complementary. Ideally,

\footnotetext{
* Based on observations collected at the European Southern Observatory, Chile, in programmes 72.C-0485, 73.C-0225, 76.C-0777, 77.C0268, 384.C-0245A.

$\star \star$ Table D.1 is also available at the CDS via anonymous ftp to cdsarc.u-strasbg.fr (130.79.128.5) or via

http://cdsarc.u-strasbg.fr/viz-bin/qcat?J/A+A/591/A84

1 See http://www. DwarfArchives .org for a full account.

2 See http://www. exoplanet. eu for further details and updates.
}

direct imaging is combined with simultaneous measurement of the radial velocity variation (Guenther et al. 2005). Young substellar objects still contract and are self-luminous (Burrows et al. 1997) so that their direct detection is less difficult up to ages of a few hundred Myr (Malkov et al. 1998; Neuhäuser \& Schmidt 2012). Unlike high-resolution imaging in space (e.g. Marengo et al. 2006), ground-based observations need to correct the seeing that can be done with adaptive optics (e.g. Neuhäuser et al. 2003; Duchêne et al. 2007). Coronagraphy with intransparent (e.g. McCarthy \& Zuckerman 2004; Chauvin et al. 2005) or semi-transparent coronagraphs (e.g. Biller et al. 2010; Boccaletti et al. 2013; Guenther et al. 2005; Itoh et al. 2006, 2008a; Neuhäuser et al. 2007; Wahhaj et al. 2011) shade the bright star so that the exposure time and thus the sensitivity of the images can be increased. A number of very sophisticated techniques have been developed recently (see the review by Fischer et al. 2014).

The frequency of low-mass companions to stars gives important clues regarding our understanding of the formation of brown 
dwarfs and planets (Ida \& Lin 2004; Alibert et al. 2005; Broeg 2007). At wide orbits, where direct imaging surveys are sensitive, the frequency of brown dwarfs is of the order of several percent for host stars with spectral types A-M and is less constrained for giant planets (Rameau et al. 2013; Bowler et al. 2015). In young nearby associations Neuhäuser \& Guenther (2004) measure a frequency of $6 \pm 4 \%$ of substellar companions which is not very different from the value of $1 \pm 1 \%$ obtained for isolated late-type stars (McCarthy \& Zuckerman 2004). The frequency of brown dwarfs around Hyades members is not very different from the latter value (Guenther et al. 2005; Bouvier et al. 2008; Lodieu et al. 2014) although the Hyades are still young at an age of $\approx 600 \mathrm{Myr}$. In the younger Pleiades (125 Myr), Yamamoto et al. (2013) confirmed two brown dwarf companions in a sample of 20 stars.

Young moving groups of an age intermediate between the Pleiades and the Hyades offer interesting opportunities to study homogeneous samples of common age and origin. No substellar companions have been found in the Her-Lyr association (Eisenbeiss et al. 2007; Biller et al. 2013) which has an age of $\approx 250$ Myr, similar to the UMa group (Eisenbeiss et al. 2013). Although a systematic survey of the UMa group at high resolution has been missing, low-mass companions have been detected: GJ 569 Ba, Bb (Martín et al. 2000; Zapatero Osorio et al. 2004), HD 130948 B \& C, (Potter et al. 2002), and $\chi^{1}$ Ori B (König et al. 2002) ${ }^{3}$. The only known planet around a probable UMa group member, $\epsilon$ Eri, was found by radial velocity variations (Hatzes et al. 2000; Benedict et al. 2006). Although there are still doubts about its existence (Zechmeister et al. 2013), there are even suggestions that a second planet might exist (Quillen \& Thorndike 2002; Deller \& Maddison 2005). The planet(s) of $\epsilon$ Eri have been subject to many attempts (still unsuccessful) of direct detection (Itoh et al. 2006; Janson et al. 2007; Marengo et al. 2006; Neuhäuser \& Schmidt 2012, and the present work).

The goal of the present work is to find additional substellar companions in the UMa group by direct imaging. The search for close and faint companions in the UMa group benefits from a relatively young age of $200-600 \mathrm{Myr}^{4}$, the small distance of members $(\approx 50$ pc on average; Fig. 1$)$, and thus the availability of precise HIPPARCOS astrometry (Perryman et al. 1997; van Leeuwen 2007). Furthermore, the proper motion of UMa group members is high on average $(\approx 150 \mathrm{mas} / \mathrm{yr})$ because of their proximity (Fig. 1) and their peculiar space motion (Ammler 2006). Hence, co-moving companions can already be identified after a short epoch difference. Preliminary results of the present study have already been published (Ammler-von Eiff et al. 2009).

\section{Coronagraphic observations and data reduction}

As the definition of the UMa group and the list of members is controversial, we compiled the targets in the following way to obtain a meaningful number of reliable UMa group

\footnotetext{
3 It is worth noting that Bannister \& Jameson (2007) identified one $\mathrm{T}$ dwarf and three $\mathrm{L}$ dwarfs in the UMa group using the moving cluster method.

4 The Ursa Major (UMa) group has been studied extensively under various aspects (e.g. Eggen 1994; Montes et al. 2001; King et al. 2003; Fuhrmann 2004; Ammler 2006) but the precise age and the list of members are still a matter of debate. Age estimates range from 200 to 600 Myr (König et al. 2002; King et al. 2003; Fuhrmann 2004; King \& Schuler 2005; Brandt \& Huang 2015).
}

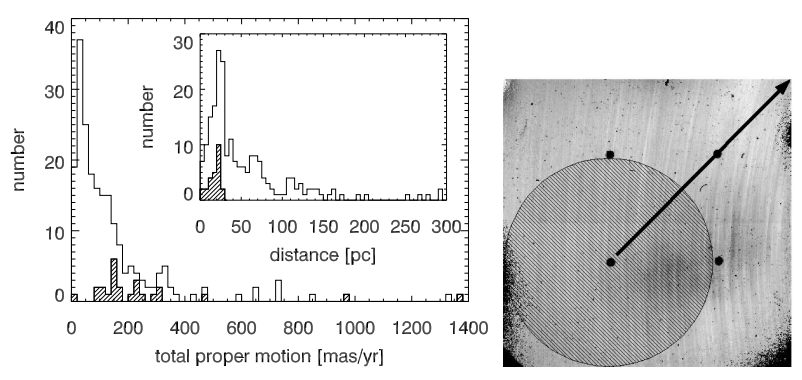

Fig. 1. Left: distribution of distance and proper motion. The major concentration of the UMa group is the UMa open cluster in the Big Dipper constellation at distances of about $25 \mathrm{pc}$. All known members and candidates (see references in the text) are included in the histogram (solid line; distances mostly from Montes et al. 2001 and van Leeuwen 2007). We add the distribution of the southern stars within $30 \mathrm{pc}$ addressed in the present work (hatched; distances from van Leeuwen 2007). Right: image of the coronagraphic mask with the four coronagraphs (black filled circles) taken with the S27 camera. Each of the coronagraphs has an angular diameter of 0.7 . We placed the star below the lower left (south-eastern) coronagraph, so that a field with a radius of $\approx 9^{\prime \prime}$ is completely covered outside the coronagraph (hatched circle). Incomplete coverage is achieved up to separations of $\approx 25^{\prime \prime}$ (arrow).

members closer than $30 \mathrm{pc}$ and observable with NACO at the ESO VLT:

- stars found by Montes et al. (2001) that fulfil at least one of Eggen's kinematic criteria (Eggen 1995);

- certain or probable members compiled by King et al. (2003) based on photometric, kinematic, and spectroscopic criteria;

- HD 135599 which is an UMa group member according to Fuhrmann (2004).

We did not observe one system, GJ 569 (=HIP 72944) because it has been extensively studied before (e.g. Simon et al. 2006). Some objects were excluded because of known bright secondaries in the field of view (HD 24916, HD 29875, HD 98712, and HD 134083). HIP $104383 \mathrm{~A}$ is also a close binary but fully fits below the coronagraph so that we did observe it (Fig. D.1), i.e. 20 targets in total (Tables A.1 and A.2).

The targets were observed with NACO, the adaptive optics imager at the Nasmyth platform of the ESO VLT UT4 (Yepun) ${ }^{5}$. The stars themselves were used as adaptive optics reference stars for the visual wavefront sensor of NAOS (VIS). First epoch images were taken in 2003/2004 (programmes 072.C-0485, 073.C-0225) and second epoch imaging followed in 2005/2006 (programmes 076.C-777, 077.C-0268) for those stars with identified faint companion candidates.

The semi-transparent coronagraph is a rarely used NACO mode. It has a diameter of 0,7 and dims the incoming stellar light by about 6 mag in the $K$ band (see Fig. 1). Hence, it blocks most of the light of the star but still allows precise astrometry with the stellar point spread function (PSF).

The observations were obtained in the $K_{\mathrm{s}}$ band (Persson et al. 1998; Tokunaga et al. 2002) further reducing the brightness difference of stars and any low-mass companions. The sensitivity of the observations has been further improved by not using the S13 camera with the smallest pixel scale but instead observing with the S27 camera (27.15 mas/pix, FOV $\left.28^{\prime \prime} \times 28^{\prime \prime}\right)$.

\footnotetext{
5 NACO consists of the adaptive optics system NAOS (Lenzen et al. 2003) and the camera CONICA (Rousset et al. 2003). The instrument was decommissioned in August 2013 and recommissioned on UT1 in January 2015.
} 
This means that a larger field is covered (Fig. 1) and more light is collected in a single pixel. The astrometric precision using the S27 camera is sufficient since the average proper motion of the UMa group of 150 mas per year (Fig. 1) corresponds to 5.5 pixels on the detector.

The data reduction follows the usual steps of sky subtraction, flat-field correction, bad pixel correction, and shift and $\operatorname{add}^{6}$. The sky was subtracted using jittered exposures of nearby sky positions free of bright stars. The flat-field correction is based on coronagraphic night-time flat-field exposures to correct for the variable transmittance of the coronagraphic substrate and standard day-time exposures to correct for pixel-to-pixel variations of the detector below the coronagraph used. Bad pixel frames were taken from standard calibrations or derived individually with ESO tools and standard twilight flat-field exposures.

\section{Identification and characterization of candidates}

The stellar PSF was subtracted in a box of $500 \times 500$ pixels centred on the star to facilitate the visual identification of faint companion candidates. The PSF was derived by calculating the average of a set of rotated frames (in steps of $2^{\circ}$ ) and using sigma-clipping to get rid of bright features, in particular the diffraction spikes. A smoothed image was subtracted to further facilitate detection (Figs. C.1-C.5) ${ }^{7}$.

We noticed that artefacts due to dust particles on the coronagraphic substrate can be mistaken for companion candidates. The flat-field correction fails to remove them since occasional displacements of the coronagraphic substrate can occur between calibration and target exposures. We removed these artefacts from the list of companion candidates guided by a visual crossmatch of the scientific exposures with the flat-field exposures. In the most extreme cases (exposures of HD 11171 and HD 22049), several tens of artefacts had to be removed.

A wide comoving companion will not change its position relative to the star, while a distant and thus non-moving background object will reflect the stellar parallactic and proper motion (Fig. 2). Our assessment of astrometric measurement uncertainties is based on Chauvin et al. (2010) who give a long-term average of the S27 pixel scale of $27.012 \pm 0.004 \mathrm{mas} / \mathrm{pix}$ and of the detector position angle (true north) of $-0.04 \pm 0.14$. In the present work, we corrected the position angles of all candidates for the mean deviation of -0.04 from the true north. From the measurements obtained by Chauvin et al. (2010), we expect uncertainties of $0.016 \mathrm{mas} / \mathrm{pix}$ on separation and 0.15 on position angle. We neglected the errors in fitting Gaussian profiles to measure the positions of the stars and the candidates. It is as precise as a few milliarcseconds and of the order of the HIPPARCos errors (Table E.2).

The instrumental magnitudes of the target stars and the candidates were all measured in apertures of 13 pixels across which is more than double the typical FWHM of the PSF. Then, the $K_{\mathrm{s}}$ band magnitude of the candidates were derived from the measured flux ratios taking into account the brightness of the target star (Table A.2) and the attenuation of the coronagraph. While the brightness of the star was measured in the reduced

\footnotetext{
6 Using scripts and tools provided by ESO, including ESO-eclipse v5.0.0, the jitter recipe (Devillard 1997, 2011), and esorex using the recipe naco_img_twflat to derive bad pixel frames.

7 Smoothing was performed by the application of a Gaussian filter (FILTER/GAUSS in ESO MIDAS version 13SEP; Banse et al. 1983; Warmels 1992) using all adjacent pixels within a radius of 18 pixels weighted by a Gaussian with a width of $\sigma=3$ pixels.
}
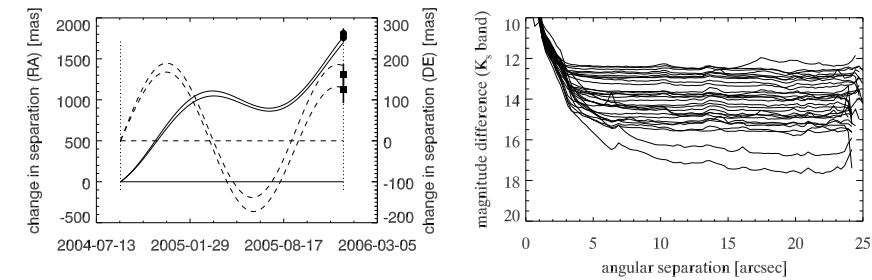

Fig. 2. Left: based on HIPPARcos data (cf. Tables E.2, A.1, and A.2), this example of HD 22049 shows how the relative motion of nonmoving background stars in right ascension (solid lines) and declination (dashed lines) will differ from a comoving companion (respective horizontal lines). The lines encompass the HIPPARCos uncertainty, scaled by a factor of 100 for visibility. Possible orbital motion of a hypothetical companion has been neglected. The vertical dotted lines highlight the dates of the 1 st and 2 nd epoch exposures, respectively. The two candidates found are background objects (circles for right ascension and squares for declination; see Table D.1 and Fig. C.1). Right: dynamic range curves for a $10 \sigma$ detection as a function of angular separation for all exposures of the survey.

frames, the brightness of the candidates was measured in the PSF-subtracted frames constructed in the way described above.

To calibrate the transmission of the coronagraph, a standard star (HD 1274) was taken during the programme 384.C-0245A, once outside and once below the coronagraph, and was reduced in the same way as the exposures of the science targets. The flux values were integrated within apertures of 19 pixels radius which fits well inside the coronagraph. Relating the measurements inside and outside the coronagraph gives a transmission of $0.47 \pm 0.03 \%$ in the $K_{\mathrm{s}}$ band which translates to a dimming of $5.83 \pm 0.06 \mathrm{mag}$. The error bar accounts for uncertainties involved in the aperture photometry but not for other systematics like cross-talk, light-leaks, temporal variation of the PSF, improper/variable placement of the star below the coronagraph, or variable residual absorption by the transparent substrate which carries the coronagraphs. The new measurement gives a dimming that is weaker by half a magnitude than the value given in the NACO manual ( $\left.K_{\mathrm{s}}=6.3 \pm 0.1 \mathrm{mag}\right)$, a measurement that was obtained in a similar way to the present work but using frames reduced via a different process and using peak counts instead of aperture photometry (ESO, priv. comm.). A conservative estimate of $6.1 \pm 0.3$ is adopted in the present work since the discrepancy cannot be explained with the data available.

For each of the companion candidates, the average magnitude of two epochs is adopted and the uncertainty is given by half their difference plus the square-added error bar of $0.3 \mathrm{mag}$ of the transmissivity of the coronagraph. For single-epoch images, the transmissivity is the only well-known dominant source of error.

\section{Assessment of field coverage and detection limits}

We could not take advantage of the full field of view of the NACO mode applied $\left(28^{\prime \prime} \times 28^{\prime \prime}\right)$ since the corners are affected by obstructions. Furthermore, the coronagraphs themselves are insensitive patches in the field of view.

The field of view is covered completely between angular separations of 0 '.35 (coronagraphic radius) and 9'.0 which correspond to different linear scales depending on the distance of the star. The parallaxes of the sample stars vary from 37 mas (HIP 104383) to 311 mas (HD 22049) so that the field of view covers very different parts of the stellar environments, e.g. the 
closest view in the case of $\epsilon$ Eri (=HD 22049; 1.1-29 au) and the farthest view for HIP 104383 (9.2-237 AU) (Table B.1). The expected widest bound orbit of low-mass companions has been estimated based on the linear law given in Close et al. (2003) given the central mass of the target systems.

Although the field of view is complete only up to separations of $\sim 9^{\prime \prime}$, it samples separations of up to $\sim 25^{\prime \prime}$ corresponding to as much as $\sim 650$ au in the case of HD 125451 and HIP 104383. Still the field of view never reaches the widest possible bound orbit in the present sample. We note, however, that inclination is a free parameter in this whole consideration and that measured separations are projected separations. Stars with exoplanets are known where very wide companions (up to one third of a parsec) with common proper motion have been detected (Mugrauer et al. 2014).

To understand the detection limits in the wings of the PSF, we built noise maps from the reduced and PSF-subtracted images. We measured the standard deviation at each pixel using $7 \times 7$ adjacent pixels. This value has been multiplied by the square root of the number of pixels in an aperture of 13 pixels across in order to compare to the photometric flux measurements described above.

Based on the noise analysis, the limiting magnitudes were derived relative to the magnitude of the primary star in the same way as by Brandeker et al. (2006). We found that a source can be detected at a given location if the flux measured is larger than a detection limit of $5-10 \sigma$ of the local noise level and so we adopted the conservative and common value of $10 \sigma$. The limits derived were corrected for the coronagraphic attenuation of $6.1 \mathrm{mag}$ inferred above. The right panel of Fig. 2 shows the average value of pixels at the same angular distance for each exposure.

Uncertain age is the largest contribution to the error budget when determining the mass of a companion that is still detectable. Using the stellar $K_{\mathrm{s}}$ band magnitude and the measured dynamic range, we calculated the $K_{\mathrm{s}}$ band magnitude of faint objects which could still have been detected at a given separation. The magnitudes were interpolated in evolutionary models to obtain a mass estimate. We used the COND03 models (Baraffe et al. 2003) for effective temperatures lower than $1300 \mathrm{~K}^{8}$ and DUSTY00 (Chabrier et al. 2000) models for temperatures higher than $1300 \mathrm{~K}$. For ages of $100 \mathrm{Myr}$, $500 \mathrm{Myr}$, and $1 \mathrm{Gyr}$, this temperature corresponds to a mass of 10,31 , and $42 M_{\text {Jup }}$, respectively, and is accessible to the present survey. In this range of mass, the difference in $K$ band magnitude between $100 \mathrm{Myr}$ and $1 \mathrm{Gyr}$ varies from 4 at the high-mass end to 8 at the low-mass end. Even if we avoid interpolating the true age range of the UMa group in the evolutionary models, we can assess that the age uncertainty of the UMa group implies an error of several $K$ band magnitudes which is the dominant source of error in the present study.

\section{Results and discussion}

The noise level is observed to decrease strongly with increasing separation from the primary star (Fig. 2). The longest ontarget exposure time was spent on the brightest target, HD 22049, resulting in the highest dynamic range of the present work

\footnotetext{
8 According to Baraffe et al. (2003), the COND03 models are more appropriate below $1300 \mathrm{~K}$ for predicting infrared colours, for methane dwarfs and extrasolar giant planets at large orbital separation. The difference between the $K_{\mathrm{s}}$ and the $K$ band has been neglected in the present study.
}
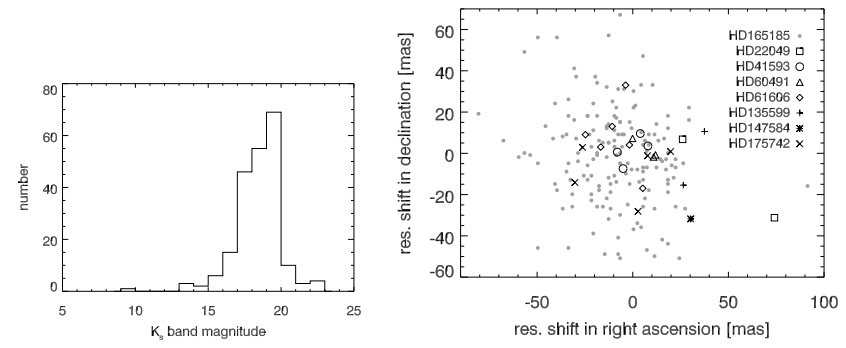

Fig. 3. Left: $K_{\mathrm{s}}$ magnitude distribution of candidates. Based on Table D.1. Errors in magnitude are not considered. Right: residual motion of candidates. For all candidates detected in two epochs, the figure shows the residual shifts in right ascension and declination over the time elapsed between the epochs (Table D.1) after correcting for the proper and parallactic motion of the central stars (Table E.2). The symbols group the candidates according to their central stars. Error bars have been omitted for clarity.

( $\gtrsim 17$ mag for a $10 \sigma$ detection). Typically, the dynamic range for a $10 \sigma$ detection limit ranges between $\Delta K_{\mathrm{s}}=13$ and 15 at a separation beyond $3^{\prime \prime}$ (Figs. 2, E.1-E.3). Given the $K$ band magnitude of the central star, this corresponds to about $12-20 M_{\text {Jup }}$ if an age of $500 \mathrm{Myr}$ is assumed (Figs. E.4 and E.5). Objects with $12 M_{\text {Jup }}$ and younger than $1 \mathrm{Gyr}$ could have been detected at separations of less than 10 au in the case of HIP 57548 which has the deepest exposure in the present work, and objects with $20 M_{\text {Jup }}$ closer than 3 au (Table E.1).

More than 200 candidates have been identified (Figs. C.1C.5, Table D.1). In some fields, not a single object has been detected around the star (HD 11171, HD 26923, HD 38393, HD 63433, HIP 57548, HD 95650, HD 125451, HD 139006, and HD 217813): however, some 160 have been found around HD 165185 which is located in the galactic plane. A number of additional candidates have been found close to HD 11131, HD 22049, HD 26913, HD 41593, HD 60491, HD 61606 A, HD 135599, HD 147584, HD 175742, and HIP $104383 \mathrm{~A}^{9}$.

The relative shift of a companion with respect to the star was measured when a second epoch was available (Table D.1). These cases are all non-moving background stars when compared to the predicted shift due to the stellar parallactic and proper motion (Table E.2 and Fig. 3) ${ }^{10}$. The deviation from these expectations is less than $00^{\prime \prime} 1$ in all cases and of the order of the astrometric error bars (Table D.1). This shows that the true astrometric uncertainties agree with the assessments by Chauvin et al. (2010) implemented here (Sect. 3).

The detection limits can be compared to the brightness of faint background objects detected in the field of view. The uncertainties of the photometric measurements are below 1 mag in almost all cases. Usually, they are close to $0.5 \mathrm{mag}$ and they increase towards the detection limit while the photometric measurements deviate by less than a magnitude from epoch to epoch. Enhanced error bars can be ascribed to smearing, noise, flux

9 HIP $104383 \mathrm{~A}$ is a special case since it appears as a visual binary below the coronagraph (Fig. D.1). According to Balega et al. (2004), it is a binary with a separation of 0 .'3 and a magnitude difference of 1.67 in the $R^{\prime}$ band. In the $K_{\mathrm{s}}$ band we measured a magnitude difference of 0.45 . The astrometric measurements presented here were done with respect to the brighter component while the co-added signal was used for photometric measurements.

10 The parallactic motion was calculated assuming a value of the obliquity of the ecliptic plane of 23.4 and expressing the solar longitude by $L=279.697+36,000.770 T$ with the time $T$ given by Julian centuries since 1900, January 0, $12 \mathrm{~h}$ (Kovalevsky 1995). The eccentricity of Earth's orbit was neglected. 
Table 1. Identification of candidates with previous detections.

\begin{tabular}{|c|c|c|c|c|}
\hline Target/cand. & \multicolumn{2}{|c|}{ Epoch/position } & \multicolumn{2}{|c|}{ Reference/magnitude/ID } \\
\hline HD 22049 & $2002-0$ & 20 & (1) & \\
\hline ID0001 & $4^{\prime \prime} .5$ & $17 ! \prime 0$ & $K^{\prime}=17.3$ & 2 \\
\hline ID0002 & $-9^{\prime \prime} 6$ & $14^{\prime \prime 2}$ & $K^{\prime}=17.3$ & 1 \\
\hline HD 41593 & \multicolumn{2}{|c|}{$2002-2004$} & (2) & \\
\hline ID0002 & $11 " ! 10$ & $328: 40$ & $H=15.88$ & $\operatorname{cc} 1$ \\
\hline HD 175742 & \multicolumn{2}{|c|}{$2004-06-28$} & \multicolumn{2}{|l|}{ (3) } \\
\hline ID0001 & $9^{\prime \prime} .45$ & 308.5 & $K_{\mathrm{s}}=16.99 \pm 0.09$ & 3 \\
\hline ID0003 & $7 \prime .57$ & 335.5 & $K_{\mathrm{s}}=19.13 \pm 0.23$ & 4 \\
\hline ID0004 & $2 " .64$ & $89^{\circ}$ & $K_{\mathrm{s}}=16.88 \pm 0.09$ & 1 \\
\hline ID0006 & $9 ! 36$ & $199^{\circ}$ & $K_{\mathrm{s}}=17.34 \pm 0.09$ & 2 \\
\hline HD 175742 & \multicolumn{2}{|c|}{$2011-05-23$} & (4) & \\
\hline ID0004 & $1 " \prime 72$ & $1 " .97$ & ... & $\ldots$ \\
\hline
\end{tabular}

Notes. For each target with previous imaging, the candidates recovered are listed and the corresponding previous astrometric and photometric measurements are given together with the reference ID assigned in the previous work. Relative positions in right ascension and declination are given for Macintosh et al. (2003) (1); and Janson et al. (2013) (4); while total separation and position angle are given for Itoh et al. (2008b) (2); and Metchev \& Hillenbrand (2009) (3).

missed by the aperture photometry at the edges of the frame, or background features like diffraction spikes, ghosts, and reflections (indicated in Table D.1).

The faintest object identified (HD 165185, ID0101) advocates an empirical detection limit of $K_{\mathrm{s}} \approx 22$ for HD 165185. However, the aperture photometry of this object is affected by insufficient background correction so that it must be somewhat brighter (Fig. C.3). Moreover, the lower envelope to the distribution of most candidates (Fig. E.2) is a bit higher as is also indicated by the brightness distribution of all candidates (cf. Fig. 3). It covers magnitudes in the range $K_{\mathrm{s}}=13-23$ with a single brighter object next to HD 11131. As can be expected for a distribution of field stars, there are a few bright objects and a larger number of faint objects. The number of objects decreases rapidly at a brightness level fainter than $K_{\mathrm{s}}=20$ indicating that the census is complete down to this value. Since the distribution is almost entirely made up of objects next to HD 165185, this completeness limit is certainly valid for this subset.

We compared our candidates with previous work for targets in common with other surveys which have not necessarily been dedicated to the UMa group (Macintosh et al. 2003; Itoh et al. 2008b; Metchev \& Hillenbrand 2009).We recovered all of the previously known candidates (Table 1) at the positions expected as long as they are covered by our field of view. Photometric measurements agree well within the error bars as long as they have been measured in the same or a similar photometric band. The single exception is ID 4 next to HD 175742 (Metchev \& Hillenbrand 2009, object 1). Most certainly, the difference of one $K_{\mathrm{s}}$ magnitude can be explained by the location of ID 4 in the PSF of HD 175742 where the photometric measurement is very sensitive to imperfections of the PSF subtraction. The same object was found by Janson et al. (2013) but no photometric measurement is given there. The southern component of HD 165185 listed by Mason et al. (2014) is out of the field of view of the present work but is listed in Table B.1.

The deepest exposure was chosen for each target to derive a survey upper limit on the frequency of stars with a companion

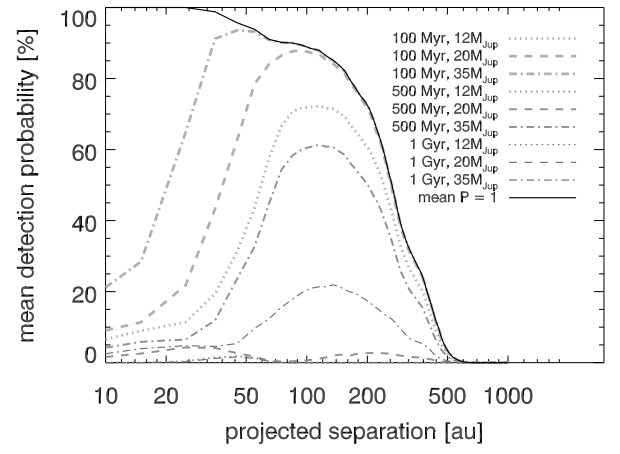

Fig. 4. Mean survey detection probability for companions of 12, 20, and $35 M_{\mathrm{Jup}}$ as a function of separation and for different age of $100 \mathrm{Myr}$, $500 \mathrm{Myr}$, and $1 \mathrm{Gyr}$ as indicated in the legend. The solid line gives the limiting case of a detection probability of 1 in each frame.

of given mass and age. This was done in a basic way similar to that used by Lafrenière et al. (2007), without assuming any prior knowledge on the mass-period distribution of companions.

For this purpose, maps of $10 \sigma$ detection probability were derived from the maps of limiting magnitude presented in Sect. 4 by comparing them to the signal of a 12,20 , and $35 M_{\text {Jup }}$ companion at different ages of $100 \mathrm{Myr}, 500 \mathrm{Myr}$, and $1 \mathrm{Gyr}$ (using the evolutionary models described in Sect. 4) ${ }^{11}$. The mean survey detection probability was assessed by centring, rescaling, and averaging these probability maps (Fig. 4). In the limiting case, where the detection probability equals 1 throughout each frame, the average detection probability is only limited by the respective field of view and approaches zero at separations beyond $\sim 500$ au, i.e. these separations are not covered by any exposure of the survey. The age range considered has a stronger effect than the mass range. On average, the survey is most sensitive at separations between 100 and 200 au since the distance of the targets and thus the field of view covered is very different.

We derived the upper limit on the frequency of stars with a companion where the mean $10 \sigma$ detection probability is high. We followed the Bayesian approach described by Lafrenière et al. (2007) using the Poisson approximation and a confidence level of $95 \%$. In the most sensitive range between 100 and $200 \mathrm{au}$, we can place an upper limit of $\sim 25 \%$ on the frequency of UMa group members with low-mass companions more massive than $35 M_{\text {Jup }}$ if the age of the UMa group is close to $500 \mathrm{Myr}$ or younger. The limit is never below $15 \%$, defined by the number of stars, the confidence level, and the case of a detection probability of one everywhere (solid line in Fig. 4).

When interpreting the outcome of the present study it is important to be aware of specific formation environments represented by the significant fraction of targets with stellar companions (cf. Tables A.2 and B.1). In addition we note that the known companions of GJ 569 have not been considered for the frequency estimate since they are closer to the star than the field covered by the present work and we did not account for the companions of HD 130948 since they have masses close to the deuterium burning limit of $12-13 M_{\text {Jup }}$ which we are not sensitive to on average. Although the results are not at variance with previous work, it is obvious that the study of the UMa group would benefit from a larger sample size. So far, northern UMa group members have not been studied systematically. These actually comprise the largest part of the UMa group. Although its densest

\footnotetext{
11 The probability has been computed that a signal exceeds the $10 \sigma$ detection threshold in the presence of noise, assuming a normal distribution.
} 
part on the northern hemisphere is within $30 \mathrm{pc}$, the group extends far beyond.

Acknowledgements. M.A. thanks Eike W. Guenther for fruitful discussions. We thank the anonymous referee for the constructive comments. A.B. acknowledges support from DFG in grants NE 515/13-1 and 13-2. M.A. was supported by a graduate scholarship of the Cusanuswerk, one of the national student elite programs of Germany, and an individual fellowship granted by the Fundação para a Ciência e a Tecnologia (FCT), Portugal (reference SFRH/BPD/26817/2006). M.A. acknowledges research funding granted by the Deutsche Forschungsgemeinschaft (DFG) under the project RE 1664/4-1. M.A. further acknowledges support by DLR under the projects 50OW0204 and 50OO1501. R.N acknowledges general support from the German National Science Foundation (Deutsche Forschungsgemeinschaft, DFG) in grants NE 515/13-1, 13-2, and 231. T.O.B.S. would like to thank Evangelisches Studienwerk e. V. Villigst, the state of Thuringia as well as DFG for support in program NE 515/30-1. R.N. and R.E. would like to thank DFG for support in the Priority Programme SPP 1385 on the First ten Million years of the Solar System in project NE 515/34-1 and 34-2. Use was made of the CDS services SIMBAD, Vizier, and the NASA/ADS abstract service. This research has made use of the Washington Double Star Catalog maintained at the US Naval Observatory. This publication makes use of data products from the Two Micron All Sky Survey, which is a joint project of the University of Massachusetts and the Infrared Processing and Analysis Center/California Institute of Technology, funded by the National Aeronautics an Space Administration and the National Science Foundation.

\section{References}

Alibert, Y., Mordasini, C., Benz, W., \& Winisdoerffer, C. 2005, A\&A, 434, 343 Allende Prieto, C., \& Lambert, D. L. 1999, A\&A, 352, 555

Ammler, M. 2006, Ph.D. Thesis, Astrophysikalisches Institut und Universitätsternwarte Jena, Germany

Ammler-von Eiff, M., \& Guenther, E. W. 2009, A\&A, 508, 677

Ammler-von Eiff, M., Bedalov, A., Mugrauer, M., Neuhäuser, R., \& Guenther, E. 2009, in Proc. of the 15th Cambridge Workshop on Cool Stars, Stellar Systems and the Sun, St. Andrews (Scotland), 21-25 July 2008, AIP Conf. Proc., 1094, 828

Balega, I., Balega, Y. Y., Maksimov, A. F., et al. 2004, A\&A, 422, 627

Bannister, N. P., \& Jameson, R. F. 2007, MNRAS, 378, L24

Banse, K., Crane, P., Ounnas, C., \& Ponz, D. 1983, in Proc. of DECUS, Zurich, 87

Baraffe, I., Chabrier, G., Barman, T. S., Allard, F., \& Hauschildt, P. H. 2003, A\&A, 402, 701

Benedict, G. F., McArthur, B. E., Gatewood, G., et al. 2006, AJ, 132, 2206

Biller, B. A., Liu, M. C., Wahhaj, Z., et al. 2010, ApJ, 720, L82

Biller, B. A., Liu, M. C., Wahhaj, Z., et al. 2013, ApJ, 777, 160

Boccaletti, A., Lagrange, A.-M., Bonnefoy, M., Galicher, R., \& Chauvin, G. 2013, A\&A, 551, L14

Bonavita, M., \& Desidera, S. 2007, A\&A, 468, 721

Bouvier, J., Kendall, T., Meeus, G., et al. 2008, A\&A, 481, 661

Bowler, B. P., Liu, M. C., Shkolnik, E. L., \& Tamura, M. 2015, ApJS, 216, 7

Brandeker, A., Jayawardhana, R., Khavari, P., Haisch, Jr., K. E., \& Mardones, D. 2006, ApJ, 652, 1572

Brandt, T. D., \& Huang, C. X. 2015, ApJ, 807, 58

Broeg, C. 2007, MNRAS, 377, L44

Bulut, I., \& Demircan, O. 2007, MNRAS, 378, 179

Burrows, A., Marley, M., Hubbard, W. B., et al. 1997, ApJ, 491, 856

Chabrier, G., Baraffe, I., Allard, F., \& Hauschildt, P. 2000, ApJ, 542, 464

Chauvin, G., Lagrange, A.-M., Zuckerman, B., et al. 2005, A\&A, 438, L29

Chauvin, G., Lagrange, A.-M., Bonavita, M., et al. 2010, A\&A, 509, A52

Close, L. M., Siegler, N., Freed, M., \& Biller, B. 2003, ApJ, 587, 407

Cushing, M. C., Kirkpatrick, J. D., Gelino, C. R., et al. 2011, ApJ, 743, 50

Deller, A. T., \& Maddison, S. T. 2005, ApJ, 625, 398

Devillard, N. 1997, The Messenger, 87, 19

Devillard, N. 2011, in Astrophysics Source Code Library [record ascl:1112.001]

Duchêne, G., Bontemps, S., Bouvier, J., et al. 2007, A\&A, 476, 229

Eggen, O. J. 1994, in Galactic and Solar System Optical Astrometry (Cambridge University Press), 191

Eggen, O. J. 1995, AJ, 110, 2862

Eggleton, P. P., \& Tokovinin, A. A. 2008, MNRAS, 389, 869
Eisenbeiss, T., Seifahrt, A., Mugrauer, M., et al. 2007, Astron. Nachr., 328, 521 Eisenbeiss, T., Ammler-von Eiff, M., Roell, T., et al. 2013, A\&A, 556, A53 Fabricius, C., Høg, E., Makarov, V. V., et al. 2002, A\&A, 384, 180

Fischer, D. A., Howard, A. W., Laughlin, G. P., et al. 2014, Protostars and Planets VI, 715

Fuhrmann, K. 2004, Astron. Nachr., 325, 3

Gray, D. 2005, The observation and analysis of stellar photospheres, 3rd edn. (Cambridge: Cambridge University Press)

Guenther, E. W., Paulson, D. B., Cochran, W. D., et al. 2005, A\&A, 442, 1031

Hatzes, A. P., Cochran, W. D., McArthur, B., et al. 2000, ApJ, 544, L145

Ida, S., \& Lin, D. N. C. 2004, ApJ, 604, 388

Itoh, Y., Oasa, Y., \& Fukagawa, M. 2006, ApJ, 652, 1729

Itoh, Y., Hayashi, M., Tamura, M., et al. 2008a, PASJ, 60, 223

Itoh, Y., Tamura, M., Hayashi, M., et al. 2008b, PASJ, 60, 209

Janson, M., Brandner, W., Henning, T., et al. 2007, AJ, 133, 2442

Janson, M., Brandt, T. D., Moro-Martín, A., et al. 2013, ApJ, 773, 73

King, J. R., \& Schuler, S. C. 2005, PASP, 117, 911

King, J. R., Villarreal, A. R., Soderblom, D. R., Gulliver, A. F., \& Adelman, S. J. 2003, AJ, 125, 1980

Kirkpatrick, J. D. 2008, in 14th Cambridge Workshop on Cool Stars, Stellar Systems, and the Sun, eds. S. P. P. S. D. E. M. B. J. Messina, ASP Conf. Ser., 384,85

König, B., Fuhrmann, K., Neuhäuser, R., Charbonneau, D., \& Jayawardhana, R. 2002, A\&A, 394, L43

Kovalevsky, J. 1995, Modern Astrometry (Berlin: Springer-Verlag)

Lafrenière, D., Doyon, R., Marois, C., et al. 2007, ApJ, 670, 1367

Lenzen, R., Hartung, M., Brandner, W., et al. 2003, in Instrument Design and Performance for Optical/Infrared Ground-based Telescopes. eds. M. Iye, \& A. F. M. Moorwood, SPIE Conf., 4841, 944

Lodieu, N., Boudreault, S., \& Béjar, V. J. S. 2014, MNRAS, 445, 3908

Macintosh, B. A., Becklin, E. E., Kaisler, D., Konopacky, Q., \& Zuckerman, B. 2003, ApJ, 594, 538

Malkov, O., Piskunov, A., \& Zinnecker, H. 1998, A\&A, 338, 452

Marengo, M., Megeath, S. T., Fazio, G. G., et al. 2006, ApJ, 647, 1437

Martín, E. L., Koresko, C. D., Kulkarni, S. R., Lane, B. F., \& Wizinowich, P. L. 2000, ApJ, 529, L37

Mason, B. D., Wycoff, G. L., Hartkopf, W. I., Douglass, G. G., \& Worley, C. E. 2001, AJ, 122, 3466

Mason, B. D., Wycoff, G. L., Hartkopf, W. I., Douglass, G. G., \& Worley, C. E. 2014, VizieR Online Data Catalog, B/wds

McCarthy, C., \& Zuckerman, B. 2004, AJ, 127, 2871

Metchev, S. A., \& Hillenbrand, L. A. 2009, ApJS, 181, 62

Montes, D., López-Santiago, J., Gálvez, M. C., et al. 2001, MNRAS, 328, 45

Mugrauer, M., Ginski, C., \& Seeliger, M. 2014, MNRAS, 439, 1063

Neuhäuser, R., \& Guenther, E. W. 2004, A\&A, 420, 647

Neuhäuser, R., \& Schmidt, T. 2012, in Topics in Adaptive Optics, ed. R. K. Tyson (Rijeka, Croatia: InTech)

Neuhäuser, R., Guenther, E. W., Alves, J., et al. 2003, Astron. Nachr., 324, 535

Neuhäuser, R., Mugrauer, M., Fukagawa, M., Torres, G., \& Schmidt, T. 2007, A\&A, 462, 777

Perryman, M. A. C., Lindegren, L., Kovalevsky, J., et al. 1997, A\&A, 323, L49

Persson, S. E., Murphy, D. C., Krzeminski, W., Roth, M., \& Rieke, M. J. 1998, AJ, 116, 2475

Potter, D., Martín, E. L., Cushing, M. C., et al. 2002, ApJ, 567, L133

Quillen, A. C., \& Thorndike, S. 2002, ApJ, 578, L149

Rameau, J., Chauvin, G., Lagrange, A.-M., et al. 2013, A\&A, 553, A60

Rousset, G., Lacombe, F., Puget, P., et al. 2003, in Adaptive Optical System Technologies II, eds. P. L. Wizinowich, \& D. Bonaccini, Proc. SPIE Conf., 4839, 140

Simon, M., Bender, C., \& Prato, L. 2006, ApJ, 644, 1183

Skrutskie, M. F., Cutri, R. M., Stiening, R., et al. 2006, AJ, 131, 1163

Skuljan, J., Ramm, D. J., \& Hearnshaw, J. B. 2004, MNRAS, 352, 975

Tokovinin, A., Thomas, S., Sterzik, M., \& Udry, S. 2006, A\&A, 450, 681

Tokunaga, A. T., Simons, D. A., \& Vacca, W. D. 2002, PASP, 114, 180

Valenti, J. A., \& Fischer, D. A. 2005, ApJS, 159, 141

van Leeuwen, F. 2007, A\&A, 474, 653

Wahhaj, Z., Liu, M. C., Biller, B. A., et al. 2011, ApJ, 729, 139

Warmels, R. H. 1992, in Astronomical Data Analysis Software and Systems I, eds. D. M. Worrall, C. Biemesderfer, \& J. Barnes, ASP Conf. Ser., 25, 115 Yamamoto, K., Matsuo, T., Shibai, H., et al. 2013, PASJ, 65, 90

Zapatero Osorio, M. R., Lane, B. F., Pavlenko, Y., et al. 2004, ApJ, 615, 958

Zechmeister, M., Kürster, M., Endl, M., et al. 2013, A\&A, 552, A78 


\section{Appendix A: Sample}

Table A.1. The sample of UMa group members.

\begin{tabular}{|c|c|c|c|c|c|c|c|c|c|}
\hline \multirow[t]{2}{*}{ Name } & \multicolumn{2}{|c|}{$\begin{array}{l}\text { Montes et al. } \\
\quad(2001)\end{array}$} & \multicolumn{3}{|c|}{ King et al. (2003) } & \multirow[t]{2}{*}{$\begin{array}{l}\text { Fuhrmann } \\
\text { (2004) }\end{array}$} & \multicolumn{2}{|c|}{ Proper motion [mas/yr] } & \multirow{2}{*}{$\begin{array}{r}\text { Number of } \\
\text { NACO epochs }\end{array}$} \\
\hline & $V_{\mathrm{pec}}$ & $\rho_{\mathrm{C}}$ & kin. & phot. & final & & $\mu_{\alpha} \cos \delta$ & $\mu_{\delta}$ & \\
\hline HD 11131 & $\mathrm{Y}$ & $\mathrm{Y}$ & $?$ & $Y ?$ & $Y ?$ & $\mathrm{Y}$ & $-124.54 \pm 3.03$ & $-105.82 \pm 2.93$ & 1 \\
\hline HD 11171 & $\mathrm{Y}$ & $\mathrm{Y}$ & $? / \mathrm{Y} ?$ & Y? & Y? & $\mathrm{Y}$ & $-148.11 \pm 0.25$ & $-93.43 \pm 0.24$ & 2 \\
\hline HD 22049 & $\ldots$ & $\ldots$ & $\ldots$ & $\ldots$ & $\ldots$ & $\mathrm{Y}$ & $-975.17 \pm 0.21$ & $19.49 \pm 0.20$ & 2 \\
\hline HD 26913 & $\mathrm{~N}$ & $\mathrm{Y}$ & $?$ & $?$ & $?$ & $\mathrm{Y}$ & $-102.64 \pm 0.66$ & $-113.30 \pm 0.59$ & 2 \\
\hline HD 26923 & $\mathrm{Y}$ & $\mathrm{Y}$ & $Y ?$ & $\mathrm{Y}$ & Y? & $\mathrm{Y}$ & $-109.46 \pm 0.48$ & $-108.25 \pm 0.43$ & 1 \\
\hline HD 38393 & $\mathrm{Y}$ & $\mathrm{Y}$ & $? / \mathrm{Y} ?$ & $Y ?$ & $Y ?$ & $\ldots$ & $-291.67 \pm 0.14$ & $-368.97 \pm 0.15$ & 1 \\
\hline HD 41593 & $\mathrm{Y}$ & $\mathrm{Y}$ & $\mathrm{N} ? / ?$ & $\mathrm{Y}$ & $\mathrm{N} ? /$ ? & Y & $-120.46 \pm 0.71$ & $-103.21 \pm 0.43$ & 2 \\
\hline HD 60491 & $\mathrm{~N}$ & $\mathrm{Y}$ & $\mathrm{N} ? / ?$ & $Y ?$ & $\mathrm{~N} ? / ?$ & $\ldots$ & $-81.17 \pm 1.26$ & $-42.66 \pm 0.66$ & 2 \\
\hline HD 61606 & $\mathrm{~N}$ & $\mathrm{Y}$ & $\mathrm{N} ?$ & Y? & $\mathrm{N}$ ? & $\ldots$ & $69.90 \pm 0.71$ & $-278.33 \pm 0.31$ & 2 \\
\hline HD 63433 & $\mathrm{Y}$ & $\mathrm{N}$ & $Y ?$ & $?$ & $?$ & $Y$ & $-8.31 \pm 0.65$ & $-10.48 \pm 0.46$ & 1 \\
\hline HD 95650 & $\mathrm{~N}$ & $\mathrm{Y}$ & $\mathrm{Y}$ & $Y ?$ & $\mathrm{Y}$ & $\ldots$ & $142.30 \pm 1.16$ & $-51.69 \pm 0.79$ & 1 \\
\hline HIP 57548 & $\mathrm{~N}$ & $\mathrm{Y}$ & $\mathrm{N} ?$ & $\mathrm{~N}$ ? & $\mathrm{N} ?$ & $\ldots$ & $605.26 \pm 2.32$ & $-1219.28 \pm 1.97$ & 1 \\
\hline HD 125451 & $\mathrm{~N}$ & $\mathrm{Y}$ & $\ldots$ & $\ldots$ & $\ldots$ & $\ldots$ & $105.95 \pm 0.23$ & $-31.80 \pm 0.21$ & 1 \\
\hline HD 135599 & $\ldots$ & $\ldots$ & $?$ & $\mathrm{Y}$ & $?$ & $\mathrm{Y}$ & $178.35 \pm 0.66$ & $-137.52 \pm 0.62$ & 2 \\
\hline HD 139006 & $\ldots$ & $\ldots$ & $\mathrm{Y}$ & $\mathrm{Y}$ & $\mathrm{Y}$ & $\mathrm{Y}$ & $120.27 \pm 0.19$ & $-89.58 \pm 0.20$ & 2 \\
\hline HD 147584 & $\ldots$ & $\ldots$ & $\mathrm{Y}$ & $\mathrm{Y}$ & $\mathrm{Y}$ & $\ldots$ & $199.97 \pm 0.25$ & $110.97 \pm 0.43$ & 2 \\
\hline HD 165185 & $\mathrm{~N}$ & $\mathrm{Y}$ & $\mathrm{Y}$ & $\mathrm{Y} ?$ & $\mathrm{Y}$ & $\ldots$ & $105.05 \pm 0.60$ & $7.95 \pm 0.32$ & 2 \\
\hline HD 175742 & $\ldots$ & $\ldots$ & N? & $\mathrm{Y} ?$ & $?$ & $\ldots$ & $131.31 \pm 0.49$ & $-283.72 \pm 0.63$ & 2 \\
\hline HIP 104383 & $\mathrm{~N}$ & $\mathrm{Y}$ & $\ldots$ & $\ldots$ & $\ldots$ & $\ldots$ & $-77.56 \pm 2.42$ & $-32.89 \pm 0.87$ & 2 \\
\hline HD 217813 & $\mathrm{~N}$ & $\mathrm{Y}$ & $?$ & $?$ & $?$ & $\mathrm{Y}$ & $-117.70 \pm 0.59$ & $-27.66 \pm 0.49$ & 1 \\
\hline
\end{tabular}

Notes. UMa membership information, proper motion (van Leeuwen 2007), and number of epochs of NACO observations obtained for the present work. We reiterate the assignments by Montes et al. (2001) and King et al. (2003) concerning the match of Eggen's criteria or, respectively, the decision on kinematic, photometric, and final membership ("Y" = yes; "N" = no). Uncertainty is expressed by "?" and we refer the reader to King et al. (2003) for more information. Some of the stars were not observed (zero NACO epochs taken) as is explained in the text and omitted here.

Table A.2. Basic stellar data of the sample of UMa group members.

\begin{tabular}{lrrrrlrr}
\hline \hline Object & HIP number & $\alpha(2000.0)$ & $\delta(2000.0)$ & Parallax [mas] & Spec. type & $V$ mag. & $K$ s mag. \\
\hline HD 11131 & 8486 & 014923.36 & -104212.8 & $44.32 \pm 3.02$ & G1V & 6.727 & 5.149 \\
HD 11171 & 8497 & 014935.10 & -104111.1 & $43.13 \pm 0.26$ & F3III & 4.664 & 3.872 \\
HD 22049 & 16537 & 033255.84 & -092729.7 & $310.94 \pm 0.16$ & K2V & 3.730 & 1.776 \\
HD 26913 & 19855 & 041525.79 & +061158.7 & $47.49 \pm 0.68$ & G5IV & 6.960 & 5.271 \\
HD 26923 & 19859 & 041528.80 & +061112.7 & $46.88 \pm 0.47$ & G0IV & 6.314 & 4.903 \\
HD 38393 & 27072 & 054427.79 & -222654.2 & $112.02 \pm 0.18$ & F7V & 3.586 & 2.508 \\
HD 41593 & 28954 & 060640.48 & +153231.6 & $65.48 \pm 0.67$ & K0V & 6.762 & 4.822 \\
HD 60491 & 36827 & 073426.17 & -065348.0 & $40.73 \pm 1.00$ & K2V & 8.160 & 6.019 \\
HD 61606 & 37349 & 073959.33 & -033551.0 & $70.37 \pm 0.64$ & K2V & 7.200 & 4.885 \\
HD 63433 & 38228 & 074955.06 & +272147.5 & $45.45 \pm 0.53$ & G5IV & 6.930 & 5.258 \\
HD 95650 & 53985 & 110238.34 & +215801.7 & $84.95 \pm 1.05$ & M0 & 9.690 & 5.688 \\
HIP 57548 & 57548 & 114744.40 & +004816.4 & $298.04 \pm 2.30$ & M4V & 11.080 & 5.654 \\
HD 125451 & 69989 & 141916.28 & +130015.5 & $38.32 \pm 0.28$ & F5IV & 5.400 & 4.394 \\
HD 135599 & 74702 & 151559.17 & +004746.9 & $63.11 \pm 0.70$ & K0 & 7.000 & 4.958 \\
HD 139006 & 76267 & 153441.27 & +264252.9 & $43.46 \pm 0.28$ & A0V+G5V & 2.210 & 2.206 \\
HD 147584 & 80686 & 162828.14 & -700503.8 & $82.53 \pm 0.52$ & F9V+M4V & 4.910 & 3.661 \\
HD 165185 & 88694 & 180623.72 & -360111.2 & $56.97 \pm 0.48$ & G5V & 5.949 & 4.469 \\
HD 175742 & 92919 & 185553.22 & +233323.9 & $46.74 \pm 0.85$ & K0V & 8.090 & 5.637 \\
HIP 104383 & 104383 & 210845.47 & -042536.9 & $36.92 \pm 1.53$ & K6+M1 $1^{d}$ & 9.430 & 6.398 \\
HD 217813 & 113829 & 230304.98 & +205506.9 & $40.46 \pm 0.57$ & G5V & 6.652 & 5.148 \\
\hline
\end{tabular}

Notes. All parallaxes are HIPPARcos parallaxes (van Leeuwen 2007). $K_{\mathrm{s}}$ band magnitudes were taken from 2MASS (Skrutskie et al. 2006) and are combined magnitudes for tight binaries. For spectroscopic binaries, the spectral type is displayed for both components.

References. References for spectral type. ${ }^{(a)}$ Bulut \& Demircan (2007); ${ }^{(b)}$ HD 147584: mean value of M4 of the range given by Skuljan et al. (2004) (M1V-M7V); ${ }^{(c)}$ HD 175742: SB1 (Tokovinin et al. 2006); literature not unanimous on the parameters (Eggleton \& Tokovinin 2008); ${ }^{(d)}$ HIP 104383: Balega et al. (2004). 


\section{Appendix B: Field of view and nearby visual companions}

Table B.1. Characterization of the NACO field of view according to Fig. 1.

\begin{tabular}{|c|c|c|c|c|c|c|c|c|c|c|}
\hline \multirow[t]{2}{*}{ Name } & \multicolumn{2}{|c|}{ FOV } & \multirow{2}{*}{$\begin{array}{l}\text { Max. } \\
\text { FOV } \\
{[\mathrm{au}]}\end{array}$} & \multicolumn{4}{|c|}{ Nearby stellar companions } & \multirow{2}{*}{$\begin{array}{r}\text { Central } \\
\text { mass } \\
{\left[M_{\odot}\right]} \\
\end{array}$} & \multirow[t]{2}{*}{ Ref. } & \multirow{2}{*}{$\begin{array}{l}\text { Expected approx. } \\
\text { widest bound } \\
\text { orbit [au] }\end{array}$} \\
\hline & $\begin{array}{r}\text { inner } \\
{[\mathrm{au}]}\end{array}$ & $\begin{array}{r}\text { outer } \\
{[\mathrm{au}]}\end{array}$ & & $\begin{array}{r}\# 1 \\
{[\mathrm{au}]}\end{array}$ & $\begin{array}{r}\# 2 \\
{[\mathrm{au}]}\end{array}$ & ref. & WDS entry & & & \\
\hline HD 11131 & 8.3 & 213 & 590 & 4340 & $\ldots$ & 1 & 01496-1041 & 1.00 & 6 & 5400 \\
\hline HD 11171 & 8.3 & 213 & 590 & 4340 & $\ldots$ & 1 & 01496-1041 & 1.52 & 3 & 8200 \\
\hline HD 22049 & 1.1 & 29 & 80 & & $\ldots$ & 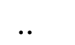 & $\ldots$ & 0.79 & 6 & 4300 \\
\hline HD 26913 & 7.3 & 188 & 522 & 1350 & $\ldots$ & 1 & $04155+0611$ & 0.96 & 6 & 5200 \\
\hline HD 26923 & 7.4 & 191 & 530 & 1360 & $\ldots$ & 1 & $04155+0611$ & 1.07 & 6 & 5800 \\
\hline HD 38393 & 3.1 & 81 & 224 & 852 & 1250 & 2 & 0544 & 1.23 & 4 & 6600 \\
\hline HD 41593 & 5.4 & 139 & 386 & $\ldots$ & $\ldots$ & .. & $\ldots$ & 0.89 & 6 & 4800 \\
\hline HD 60491 & 8.7 & 223 & 620 & & $\ldots$ & .. & $\ldots$ & 0.86 & 10 & 4600 \\
\hline HD 61606 & 5.0 & 128 & 355 & 822 & $\ldots$ & 1 & 07400-0336 & 1.43 & 5 & 7700 \\
\hline HD 63433 & 7.6 & 196 & 545 & $\ldots$ & $\ldots$ & .. & $\ldots$ & 0.99 & 6 & 5400 \\
\hline HIP 57548 & 1.2 & 30 & 83 & $\ldots$ & $\ldots$ & .. & $\ldots$ & 0.30 & 12 & 1600 \\
\hline HD 95650 & 4.1 & 105 & 292 & & $\ldots$ & .. & $\ldots$ & 0.50 & 12 & 2700 \\
\hline HD 125451 & 9.1 & 235 & 652 & 4280 & $\ldots$ & 2 & $14193+1300$ & 1.40 & 3 & 7600 \\
\hline HD 135599 & 5.5 & 140 & 389 & $\ldots$ & $\ldots$ & .. & $\ldots$ & 0.85 & 6 & 4600 \\
\hline HD 1 & 8.0 & 206 & 573 & $\ldots$ & $\ldots$ & .. & $\ldots$ & 3.50 & 11 & 18900 \\
\hline HD 147584 & 4.2 & 109 & 303 & $\ldots$ & $\ldots$ & .. & $\ldots$ & 1.39 & 8 & 7500 \\
\hline HD 165185 & 6.1 & 156 & 434 & 214 & $\ldots$ & 2 & $18064-3601$ & 1.10 & 12 & 5900 \\
\hline HD 175742 & 7.5 & 193 & 536 & $\ldots$ & $\ldots$ & .. & $\ldots$ & 1.10 & 9 & 5900 \\
\hline HD 217813 & 8.5 & 218 & 607 & $\ldots$ & $\ldots$ & .. & $\ldots$ & 1.05 & 6 & 5700 \\
\hline HIP 104383 & 9.2 & 237 & 659 & 7 & 554 & 2 & $21088-0426$ & 1.10 & 12 & 5900 \\
\hline
\end{tabular}

Notes. (2, 3) Range of separations which are completely covered around the coronograph (from inner radius, i.e. outer bound of the coronagraph of $0 \prime 35$ to the outer radius of $9^{\prime \prime}$ shown in Fig. 1). - (4) Maximum (incomplete) field of view (25"). - (5-8) Separation of up to two known nearby visual companions, reference, and WDS catalogue entry. - $(9,10)$ Mass of the central star/binary and reference. - $(11)$ Separation of the approximate widest expected bound orbit of very low-mass companions according to the approximation by Close et al. (2003) for masses greater than $0.185 M_{\odot}$. Very close (spectroscopic) components are not listed but accounted for in the central mass. Masses have been derived separately for the binary components of HIP $104383\left(0.6 M_{\odot}\right.$ and $0.5 M_{\odot}$, respectively) and then added up. HD 147584 is a spectroscopic binary with components of $1.12 M_{\odot}$ and $0.09-0.45 M_{\odot}$ (Skuljan et al. 2004, an average of $0.27 M_{\odot}$ has been adopted for the secondary). HD 175742 is a single-lined spectroscopic binary with a primary mass of $0.75 M_{\odot}$ and a minimum mass of the secondary of $0.35 M_{\odot}$ (Tokovinin et al. 2006). The maximum expected orbital separation is thus a lower limit in this particular case.

References. (1) Fabricius et al. (2002); (2) Mason et al. (2001, 2014); (3) Allende Prieto \& Lambert (1999); (4) Ammler-von Eiff \& Guenther (2009); (5) Bonavita \& Desidera (2007); (6) Fuhrmann (2004); (7) Simon et al. (2006); (8) Skuljan et al. (2004); (9) Tokovinin et al. (2006); (10) Valenti \& Fischer (2005); (11) Bulut \& Demircan (2007); (12) based on spectral type (Table A.2) and Gray (2005, Table B1). 


\section{Appendix C: Images with candidates}
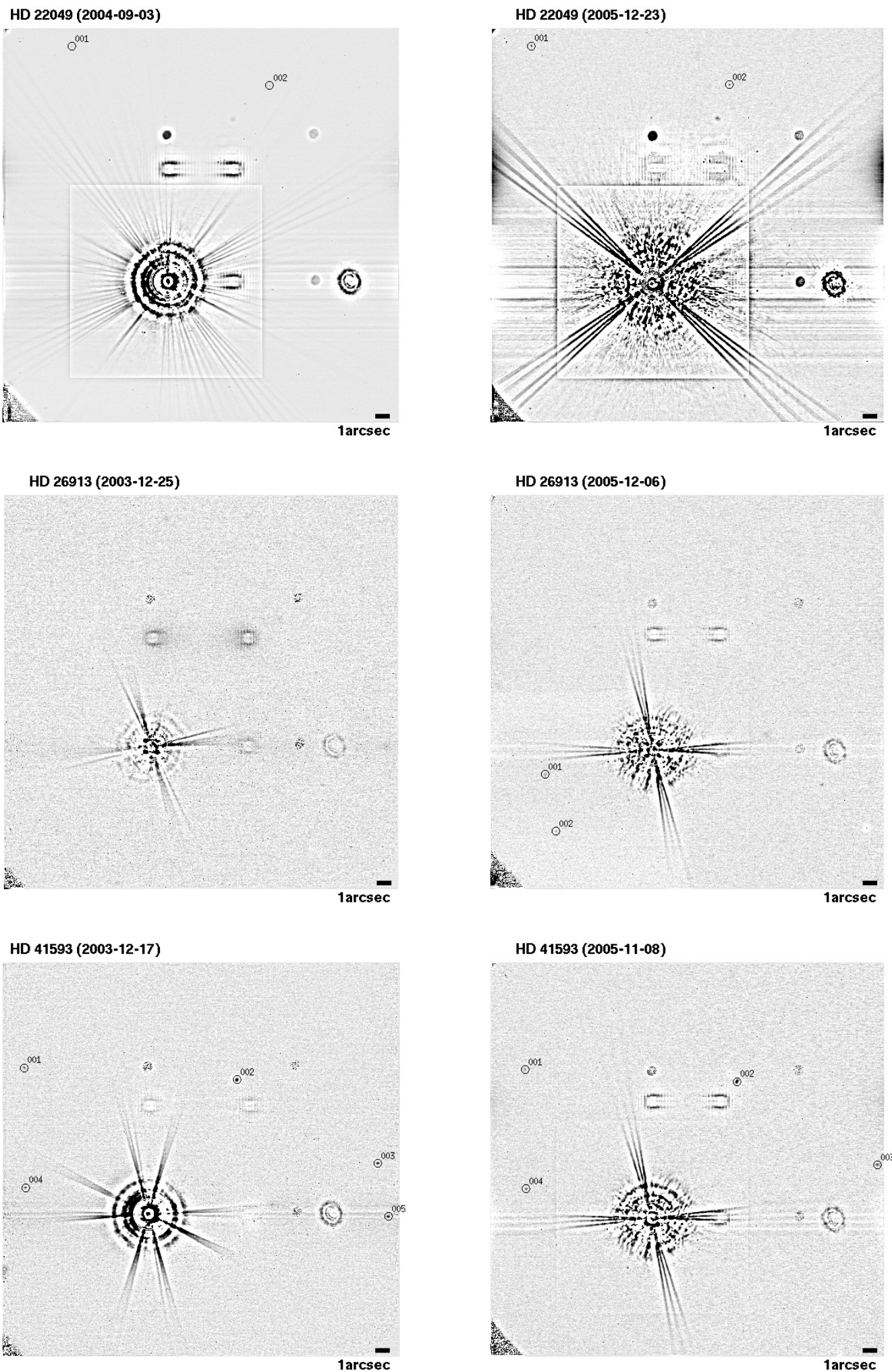

Fig. C.1. Candidates for stars with images in two epochs: HD 22049, HD 26913, and HD 41593. The images display the central star under the coronagraph. Faint companion candidates in the field of view are indicated by circles and are enumerated (cf. Table D.1). The scale is indicated in the image. North is at the top and East is to the left. The PSF of the central star has been removed in a rectangular area centred on the star and a smoothed image has been subtracted. The residual speckle pattern and the diffraction spikes of the mount of the secondary mirror remain visible (several of those due to the addition of several exposures). In addition, there are reflections, ghosts, a vignetted region to the lower left, and the shadows of the other three coronagraphs. 


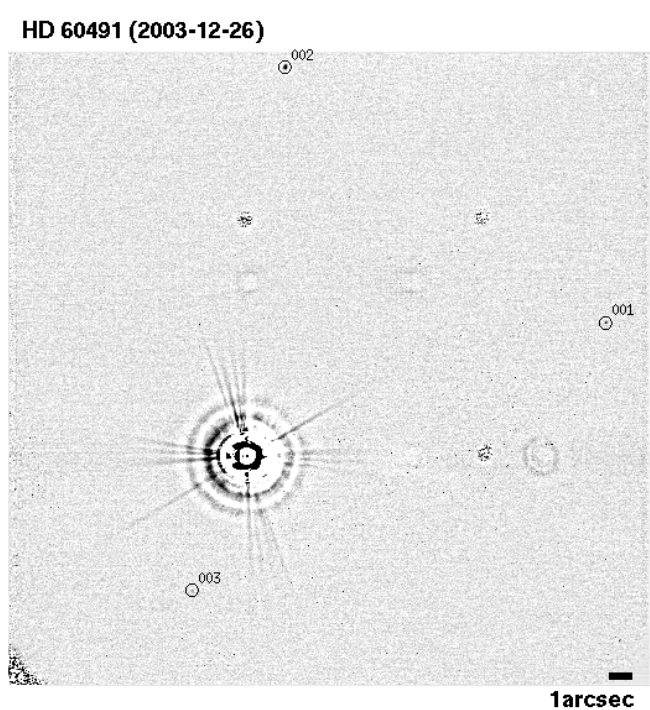

HD 61606 (2004-01-10)

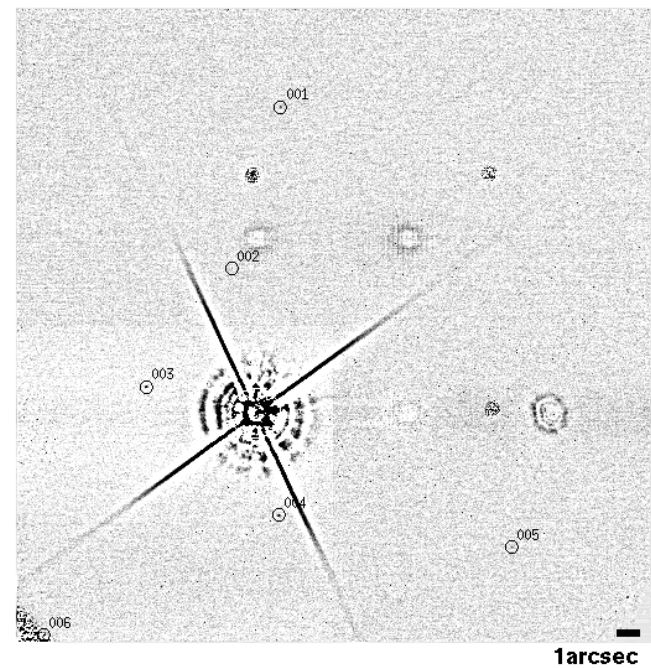

HD 135599 (2004-07-13)

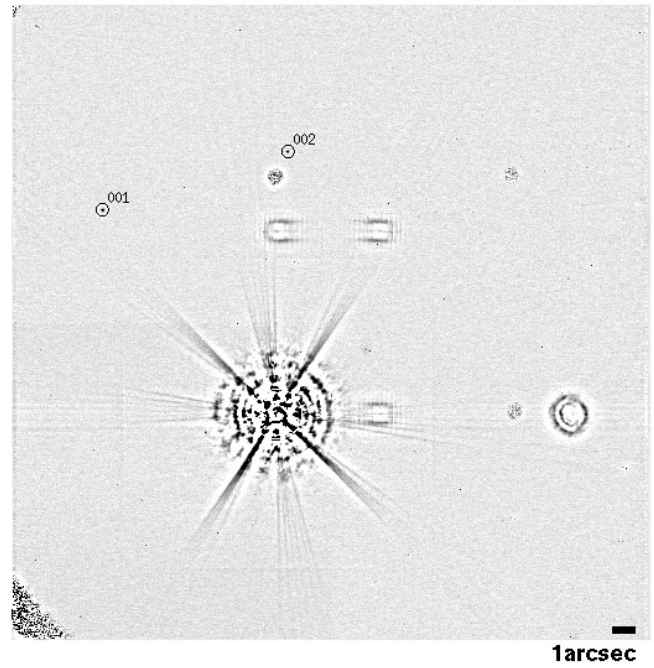

HD 60491 (2005-11-08)

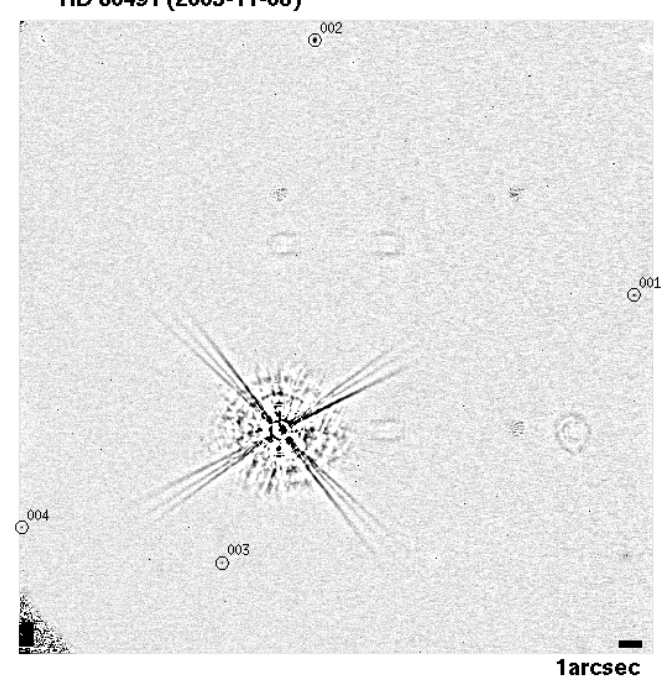

HD 61606 (2005-11-08)

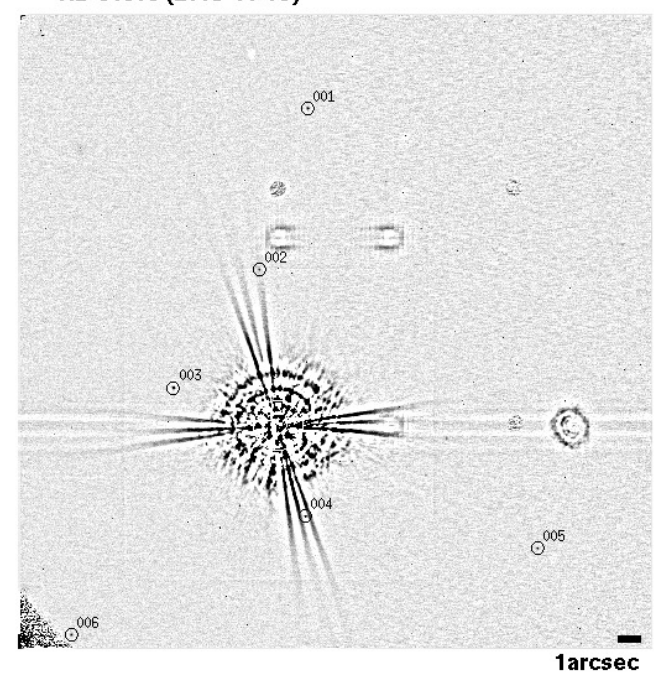

HD 135599 (2006-04-06)

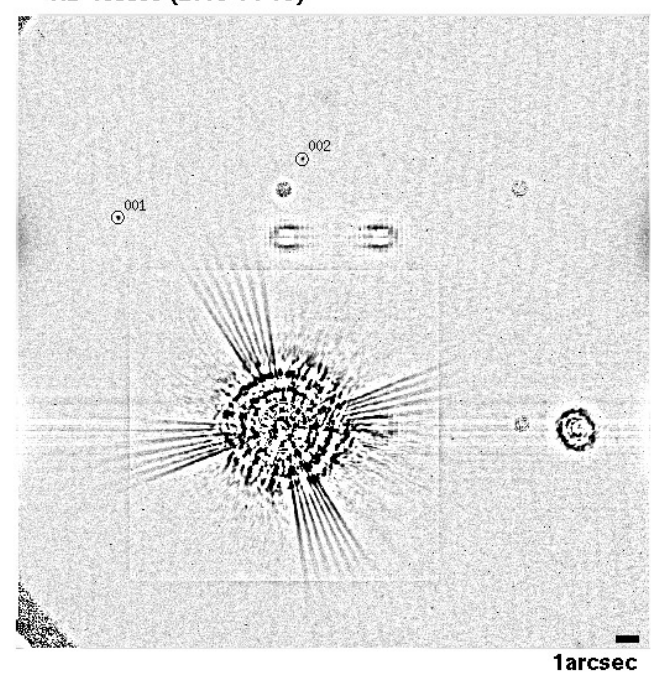

Fig. C.2. Candidates for stars with images in two epochs: HD 60491, HD 61606, and HD 135599. The layout is the same as in Fig. C.1. 
M. Ammler-von Eiff et al.: Multiplicity of UMa group members

HD 147584 (2004-02-10)
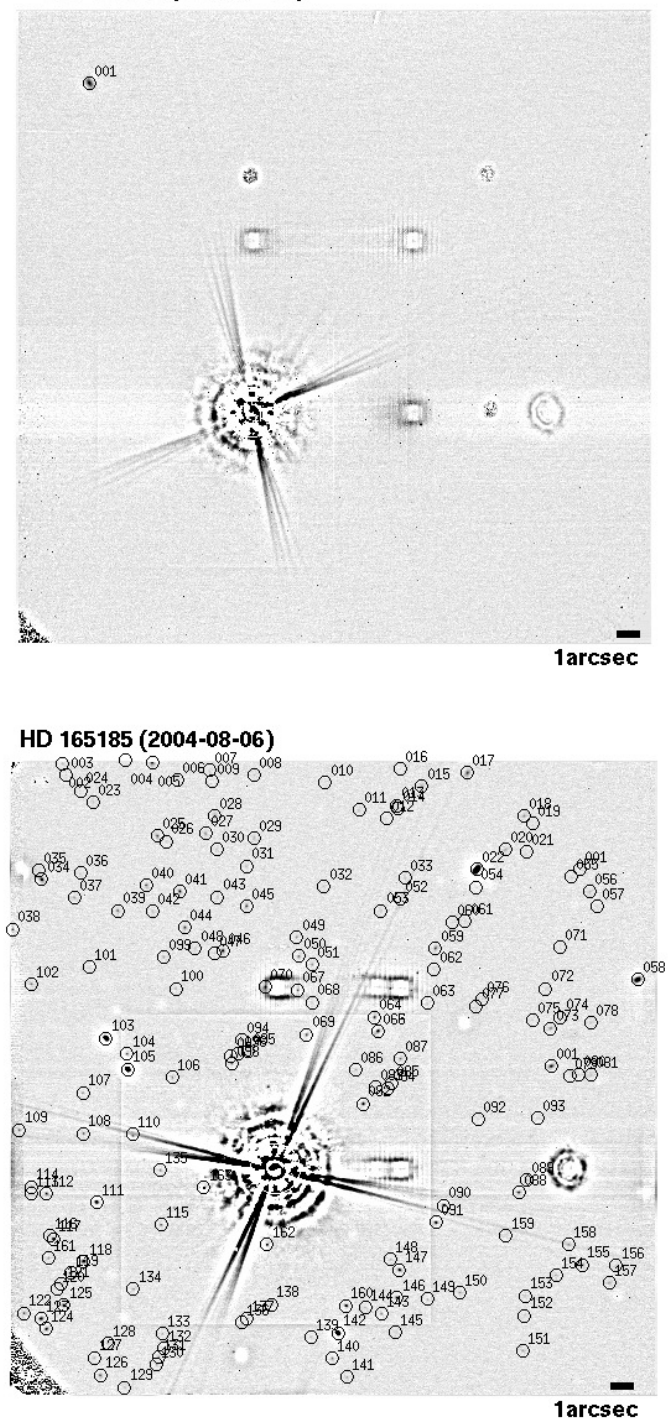

HD 175742 (2004-07-30)

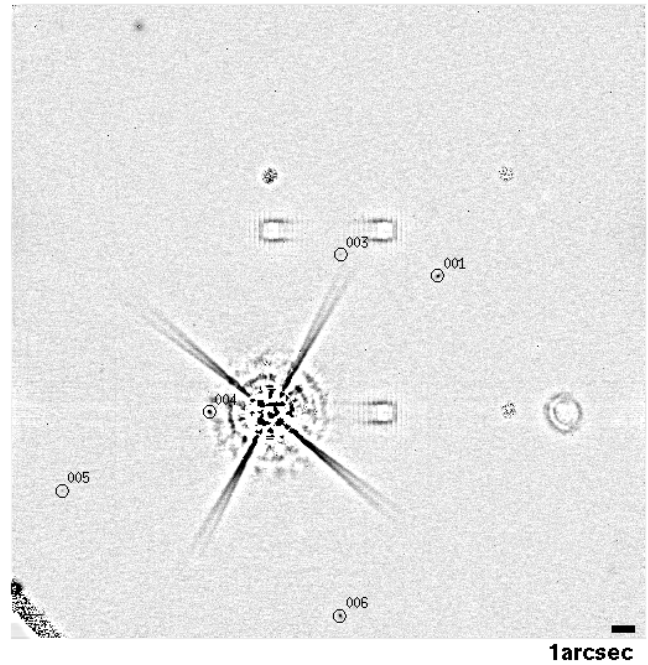

HD 147584 (2006-02-21)

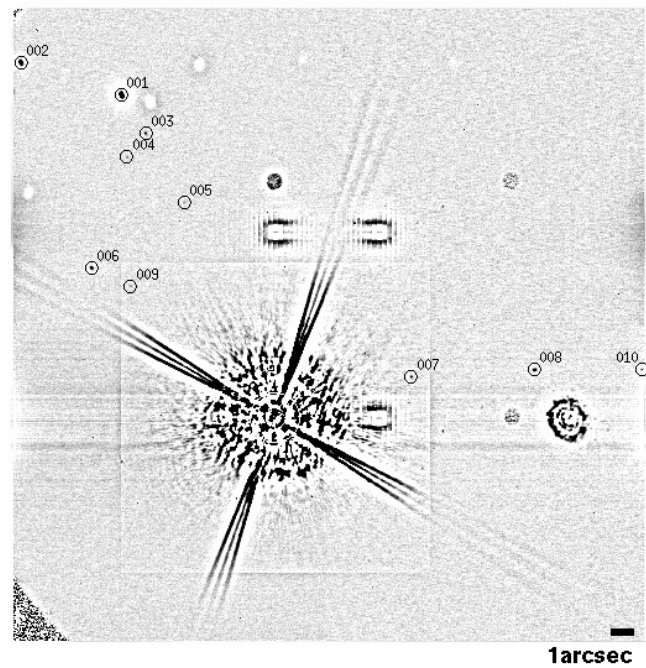

HD 165185 (2006-06-27)

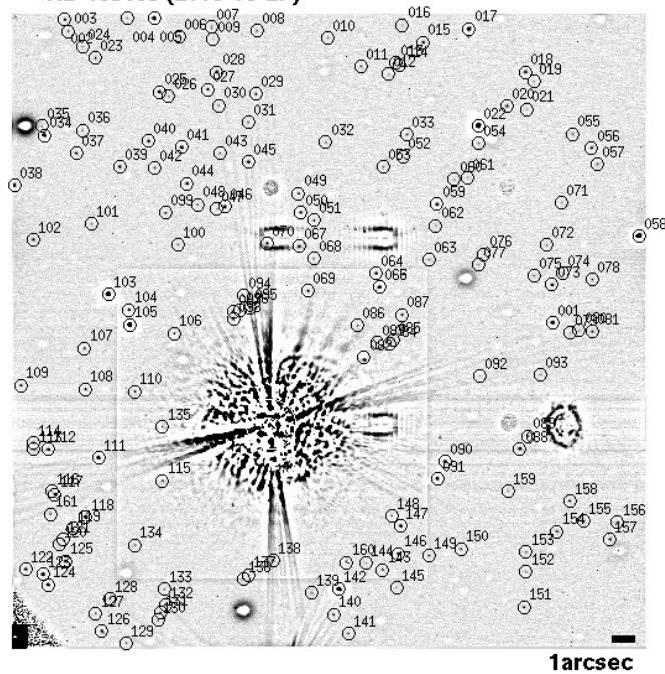

HD 175742 (2006-06-27)

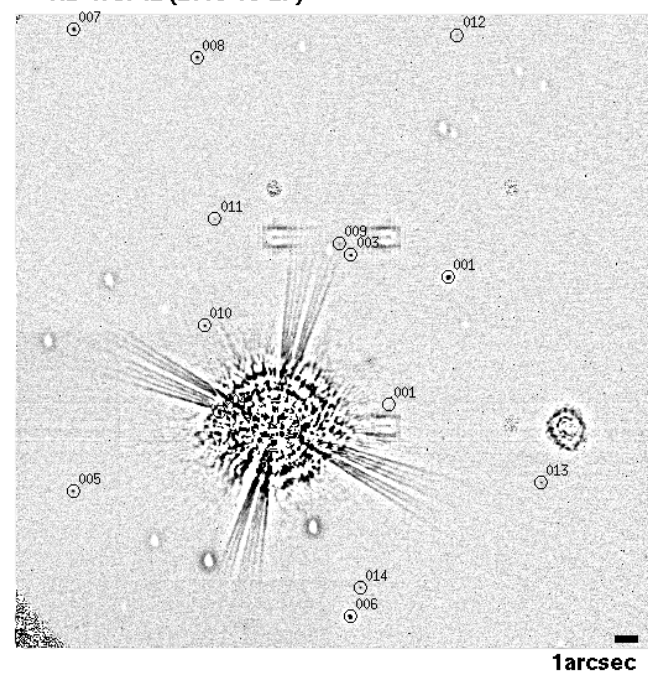

Fig. C.3. Candidates for stars with images in two epochs: HD 147584, HD 165185, and HD 175742. The layout is the same as in Fig. C.1. Stellar residuals are present in the second epochs since jittering of the sky exposures did not work in those cases. 
HD 11131 (2004-07-22)

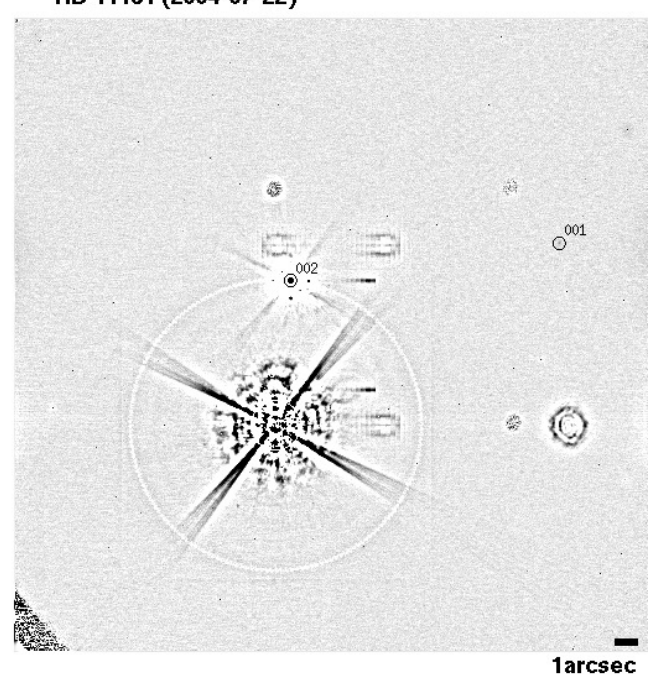

HD 11171 (2004-07-27)

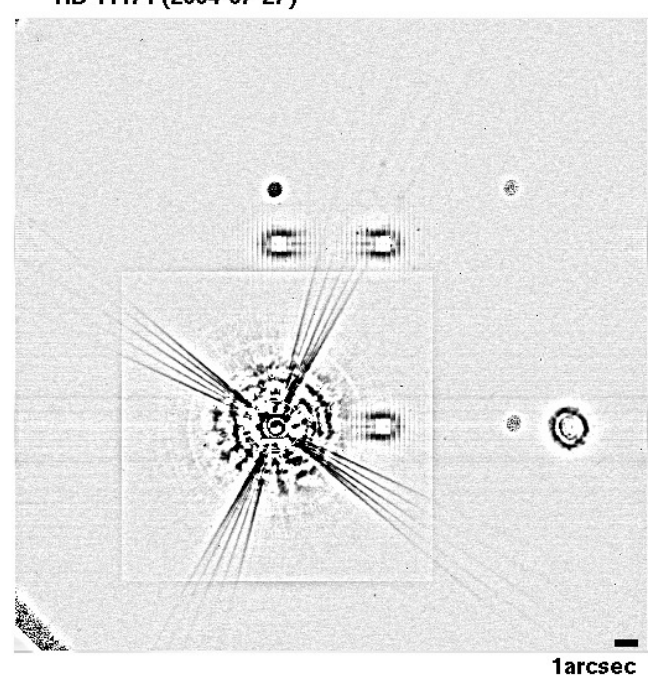

HD 26923 (2003-12-26)

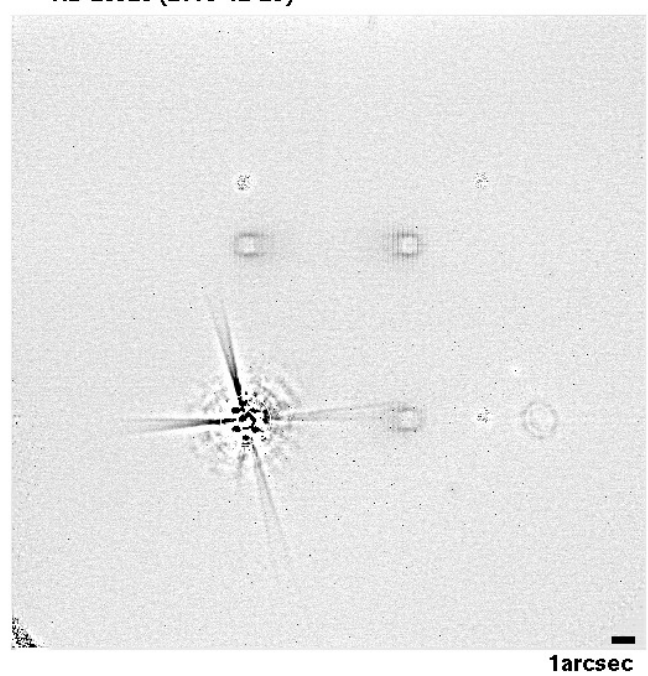

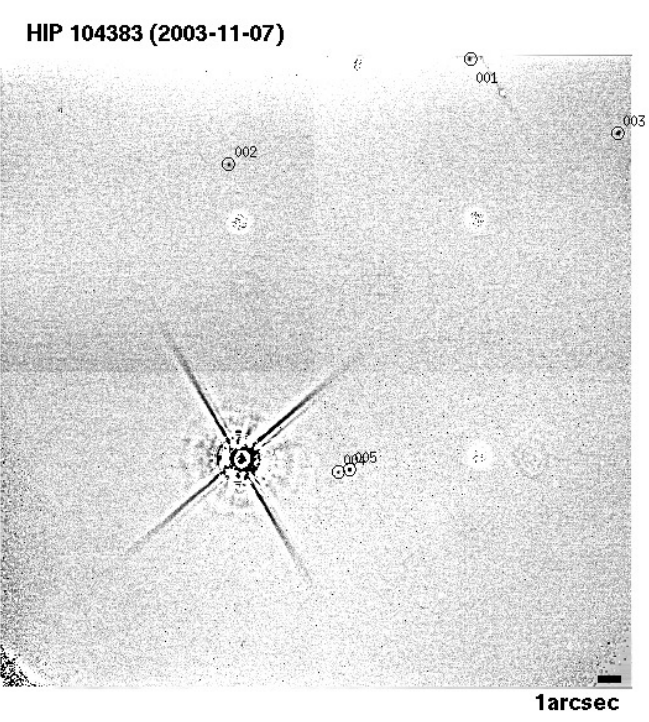

HD 11171 (2006-08-03)

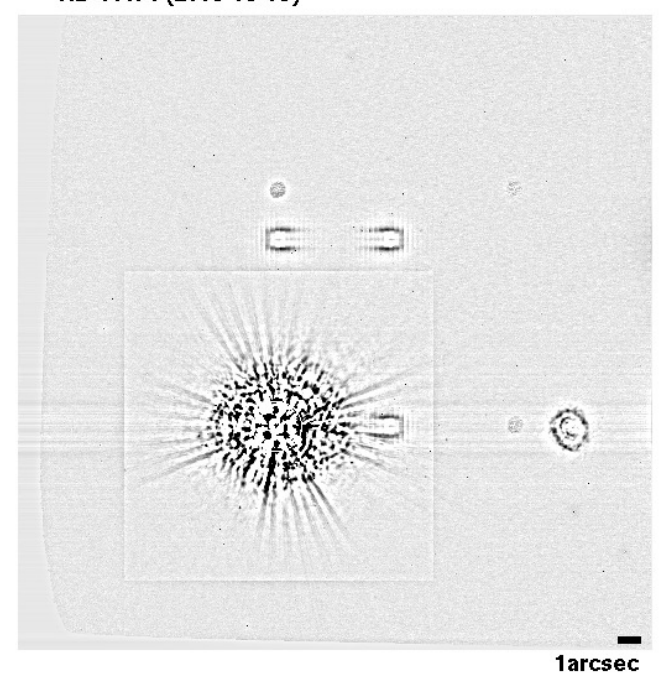

HD 38393 (2003-12-01)

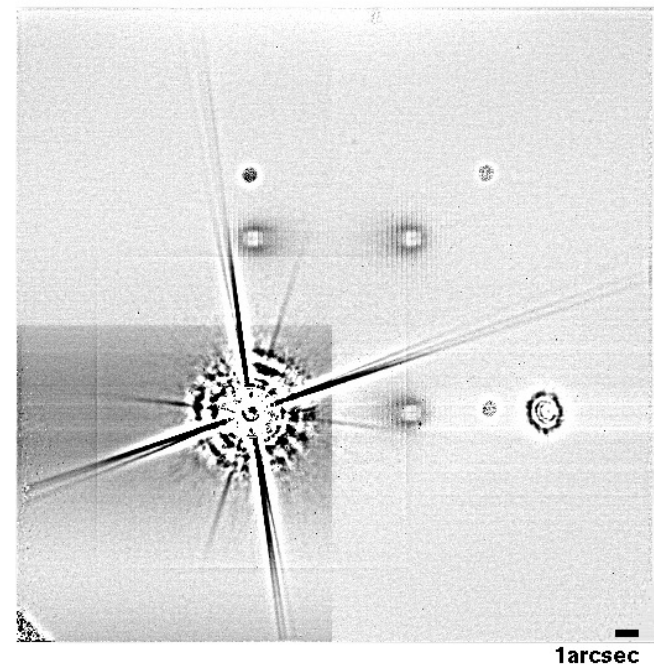

Fig. C.4. Candidates found in single-epoch observations (HD 11131 and HIP 104383) and observations of stars without any candidates (HD 11171, HD 26923, HD 38393). The layout is the same as in Fig. C.1. HIP 104383 A is a double star below the coronagraph (see Fig. D.1 and footnote to Sect. 5). 
M. Ammler-von Eiff et al.: Multiplicity of UMa group members

HD 63433 (2004-01-15)

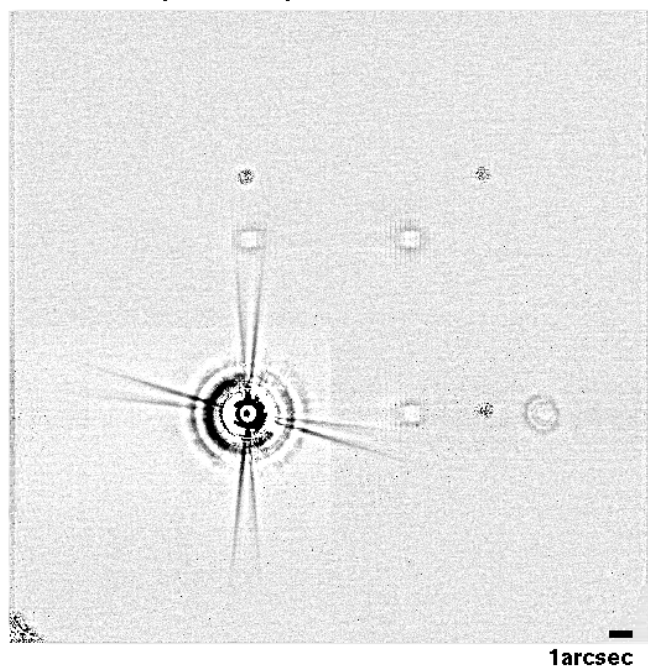

HIP 57548 (2004-01-11)

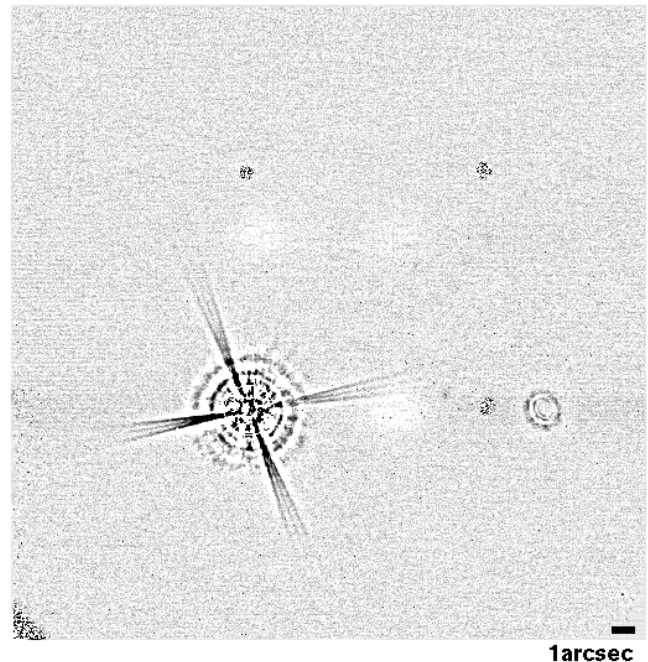

HD 139006 (2004-02-10)

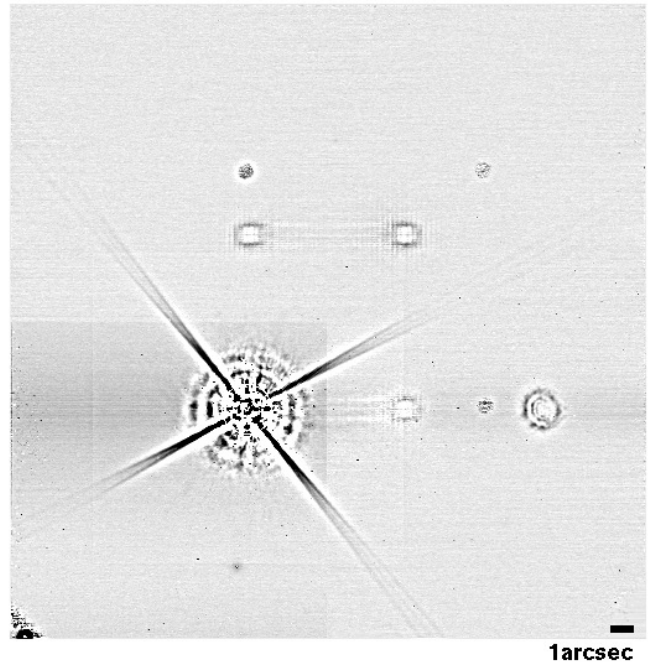

HD 95650 (2004-01-14)

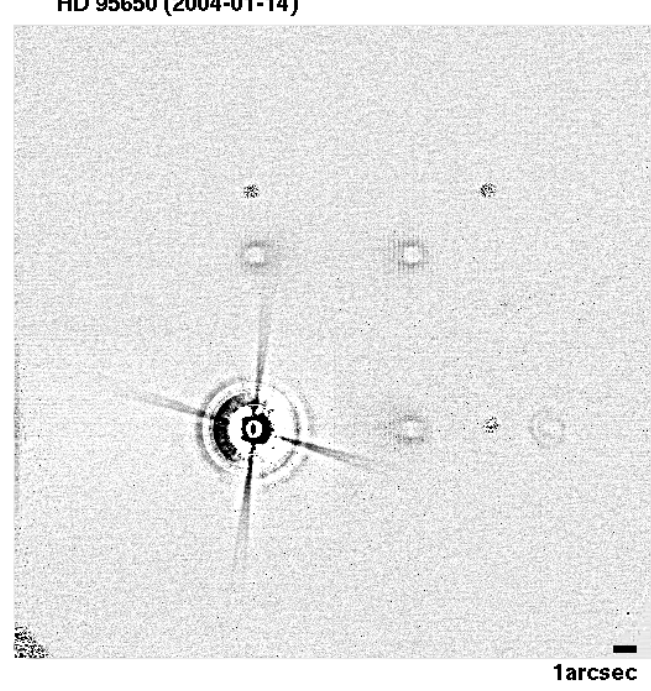

HD 125451 (2004-01-29)

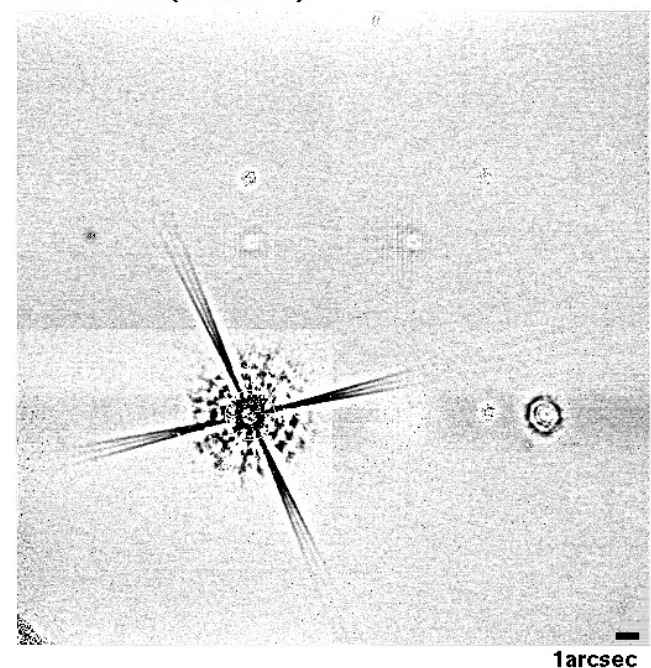

HD 217813 (2003-10-06)

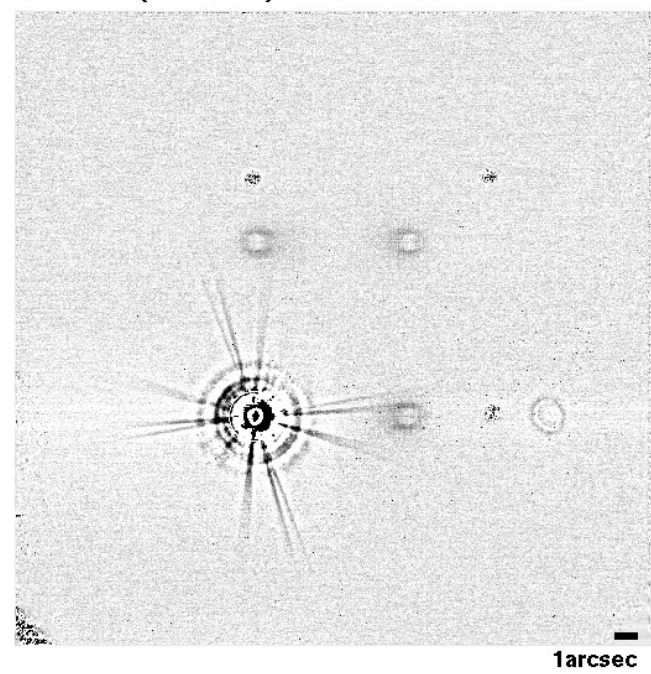

Fig. C.5. Observations of stars without any candidates: HD 63433, HD 95650, HIP 57548, HD 125451, HD 139006, and HD 217813. The layout is the same as in Fig. C.1. 


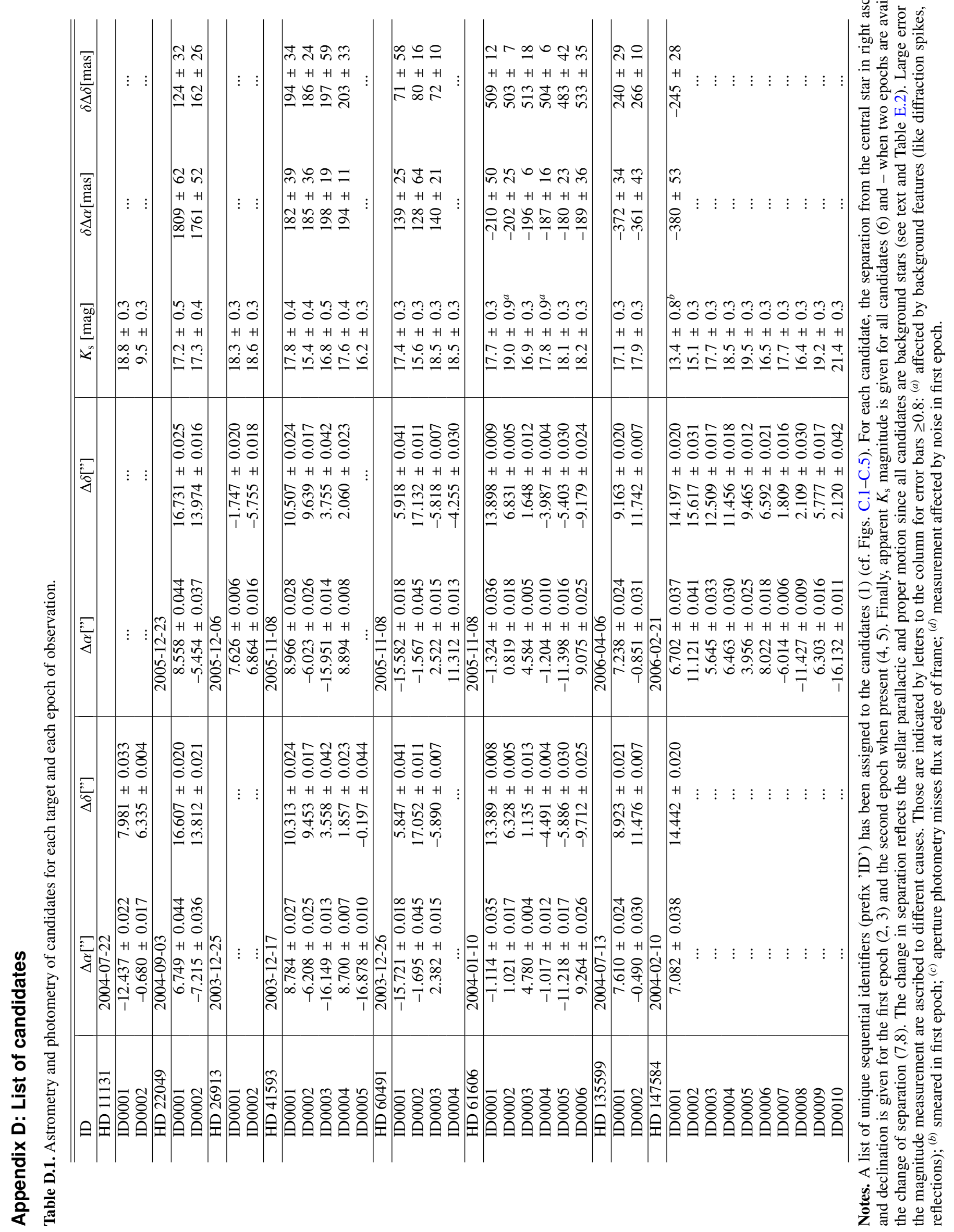




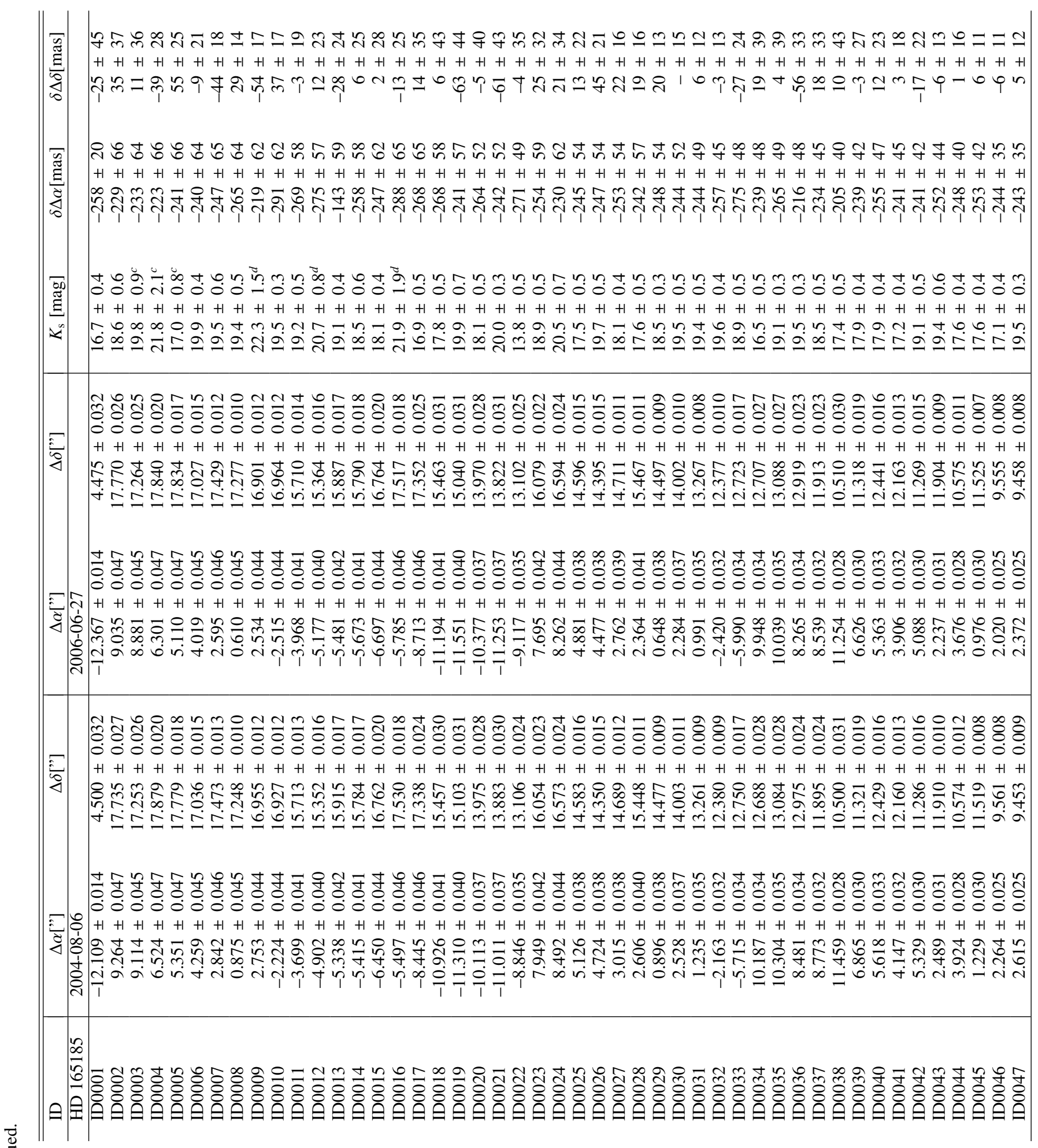




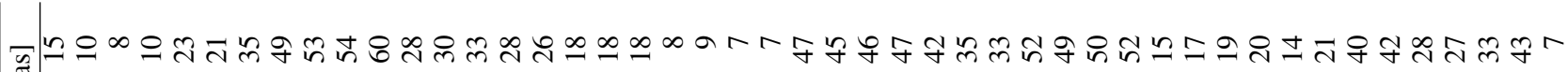
$\overparen{\Xi}+1+1+1+1+1+1+1+1+1+1+1+1+1+1+1+1+1+1+1+1+1+1+1+1+1+1+1+1+1+1+1+1+1+1+1+1+1+1+1+1+1+1+1+1+1+1+1$

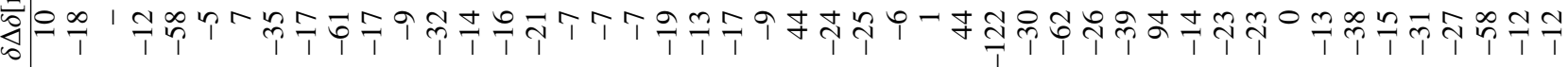

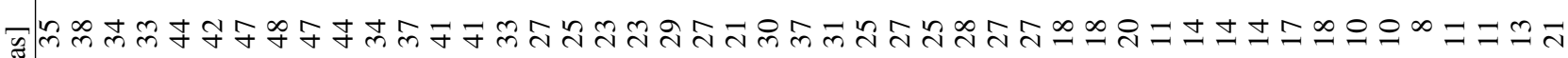
马 $+1+1+1+1+1+1+1+1+1+1+1+1+1+1+1+1+1+1+1+1+1+1+1+1+1+1+1+1+1+1+1+1+1+1+1+1+1+1+1+1+1+1+1+1+1+1+1$

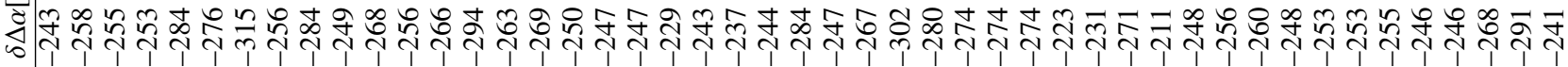

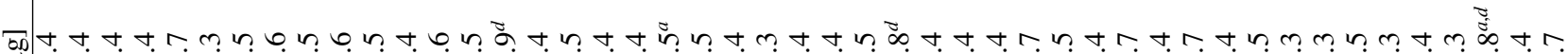

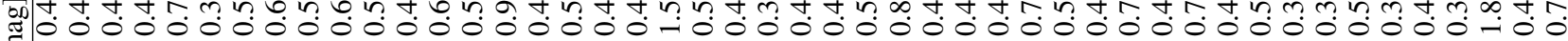
$\Xi+1+1+1+1+1+1+1+1+1+1+1+1+1+1+1+1+1+1+1+1+1+1+1+1+1+1+1+1+1+1+1+1+1+1+1+1+1+1+1+1+1+1+1+1+1+1+1$

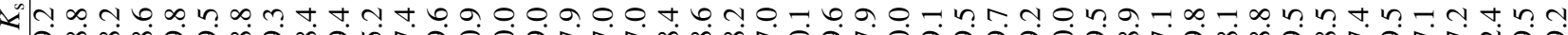

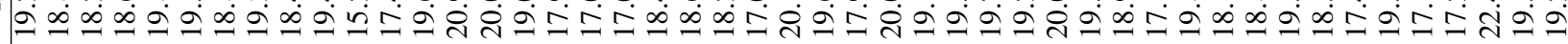

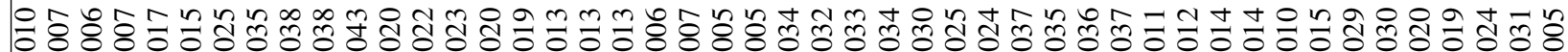
00000000000000000000000000000000000000000000000000000000000000000000 $2+1+1+1+1+1+1+1+1+1+1+1+1+1+1+1+1+1+1+1+1+1+1+1+1+1+1+1+1+1+1+1+1+1+1+1+1+1+1+1+1+1+1+1+1+1+1+1$

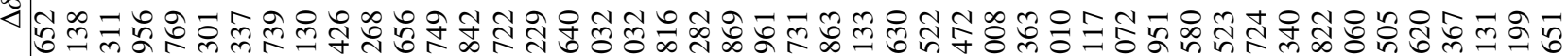

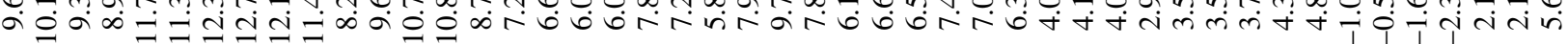

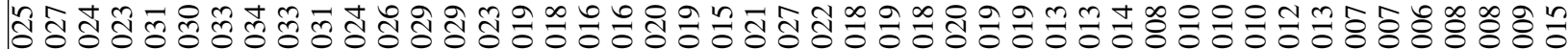

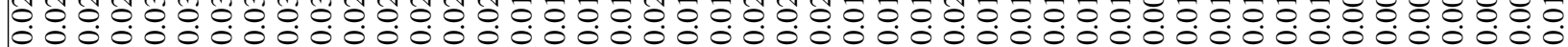
$2+1+1+1+1+1+1+1+1+1+1+1+1+1+1+1+1+1+1+1+1+1+1+1+1+1+1+1+1+1+1+1+1+1+1+1+1+1+1+1+1+1+1+1+1+1+1+1$

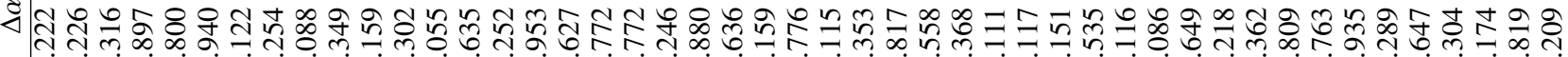

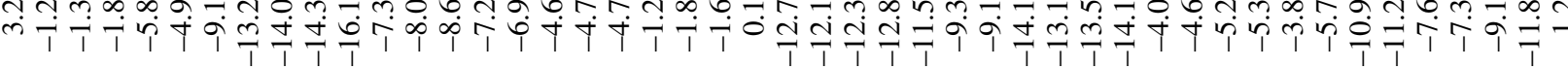

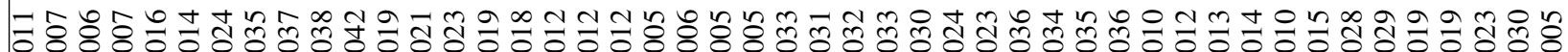

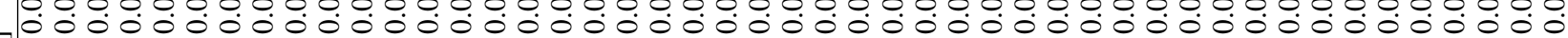
- $+1+1+1+1+1+1+1+1+1+1+1+1+1+1+1+1+1+1+1+1+1+1+1+1+1+1+1+1+1+1+1+1+1+1+1+1+1+1+1+1+1+1+1+1+1+1+1$

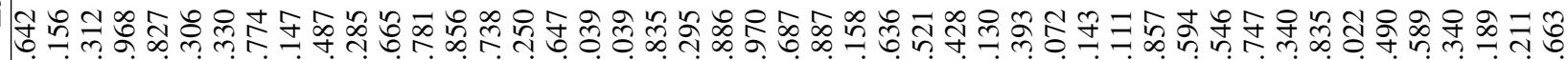

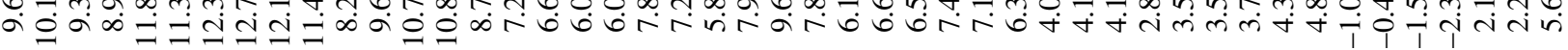

भิ讠

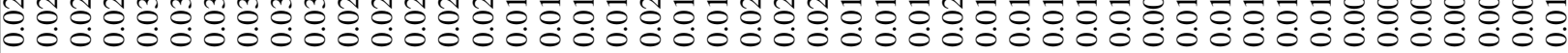
$1+1+1+1+1+1+1+1+1+1+1+1+1+1+1+1+1+1+1+1+1+1+1+1+1+1+1+1+1+1+1+1+1+1+1+1+1+1+1+1+1+1+1+1+1+1+1+1$

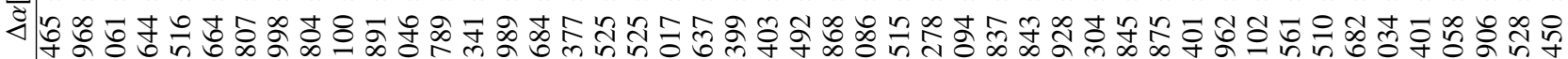

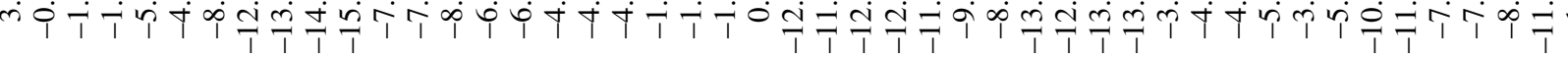




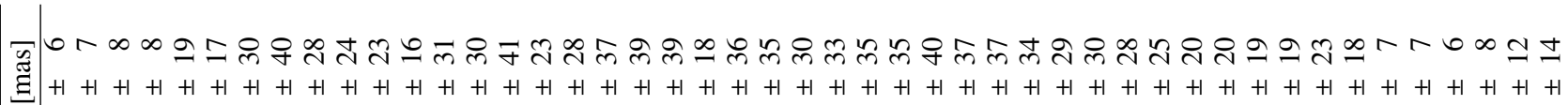

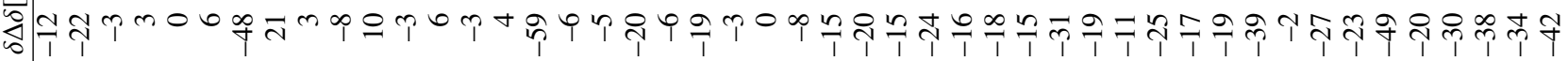

⿹⿻一𠃋小 药 $+1+1+1+1+1+1+1+1+1+1+1+1+1+1+1+1+1+1+1+1+1+1+1+1+1+1+1+1+1+1+1+1+1+1+1+1+1+1+1+1+1+1+1+1+1+1+1$

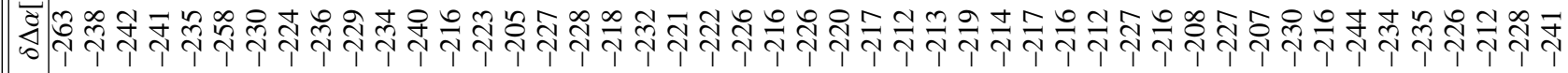

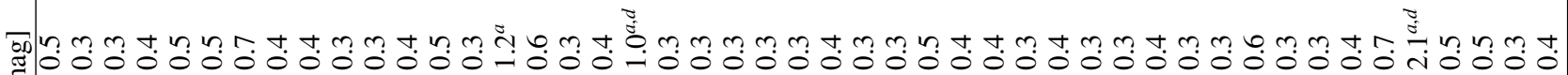

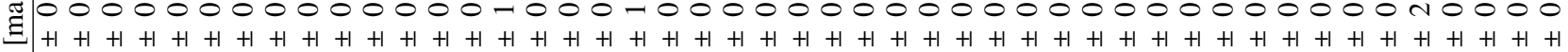

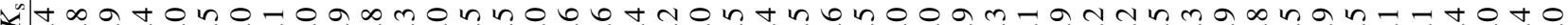

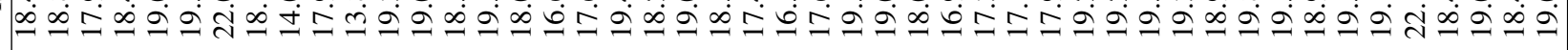

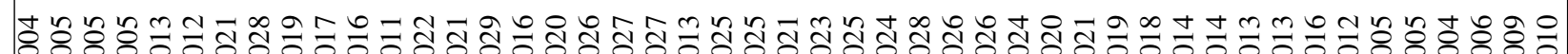
: $5+1+1+1+1+1+1+1+1+1+1+1+1+1+1+1+1+1+1+1+1+1+1+1+1+1+1+1+1+1+1+1+1+1+1+1+1+1+1+1+1+1+1+1+1+1+1+1$

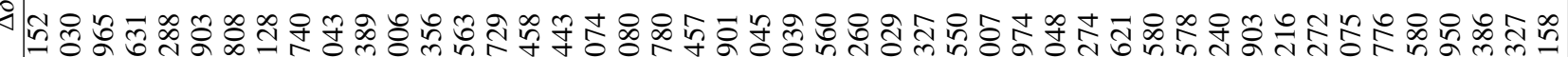

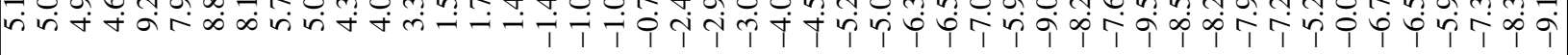

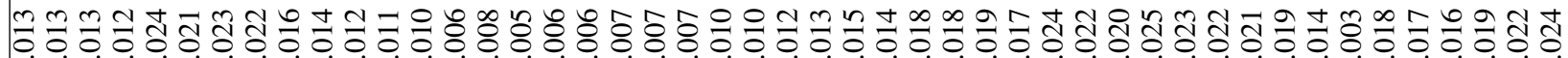
$-0.000000000000000000000000000000000000000000000$

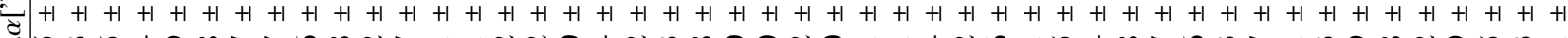

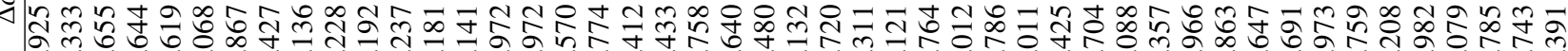

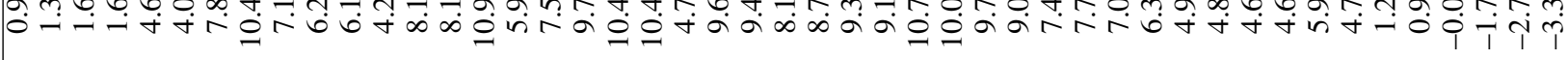

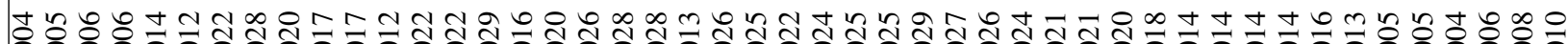

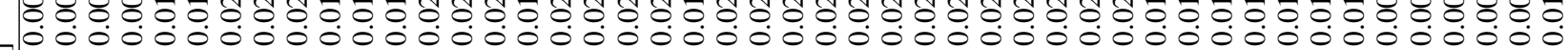
20

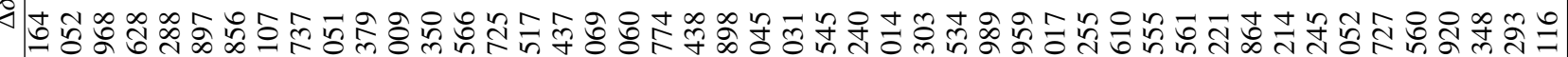

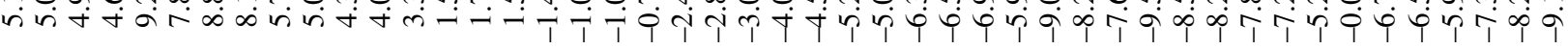

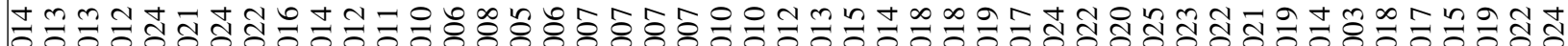

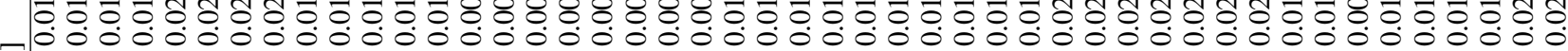
$2+1+1+1+1+1+1+1+1+1+1+1+1+1+1+1+1+1+1+1+1+1+1+1+1+1+1+1+1+1+1+1+1+1+1+1+1+1+1+1+1+1+1+1+1+1+1+1$

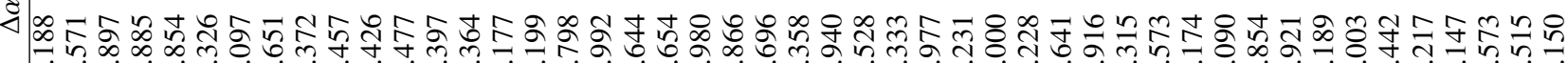

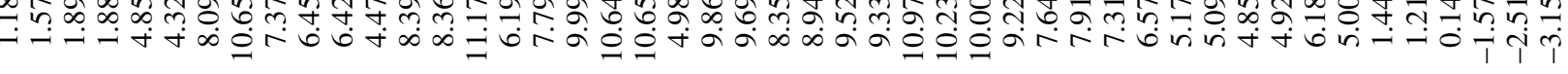

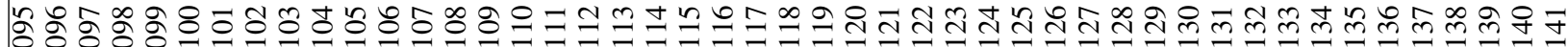

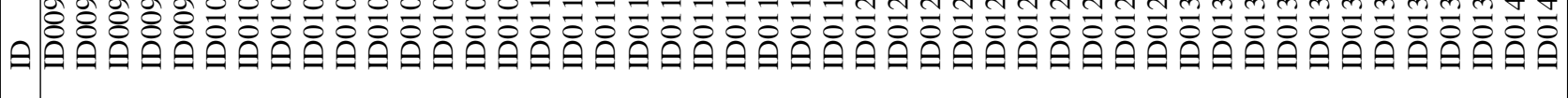




\begin{tabular}{|c|c|c|}
\hline 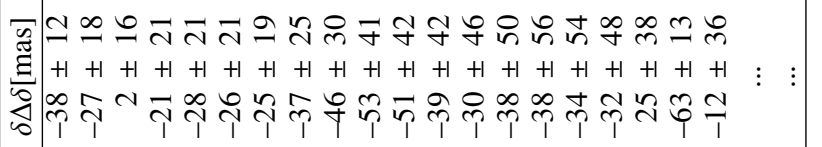 & 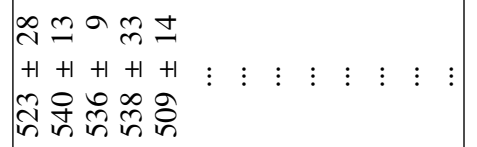 & $\begin{array}{l}\vdots \\
\vdots\end{array} \quad: \quad:$ \\
\hline 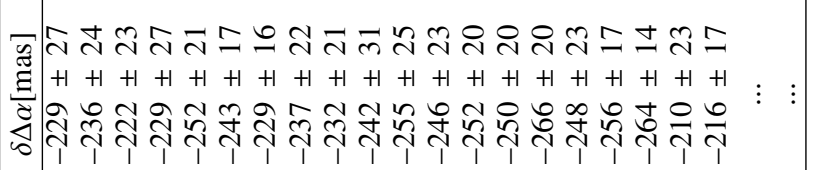 & 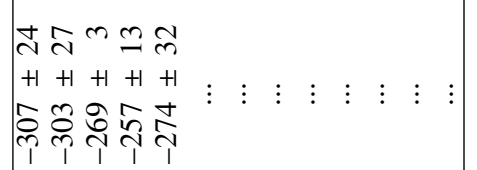 & $\begin{array}{lllll} & \vdots & \vdots & \vdots & \vdots\end{array}$ \\
\hline 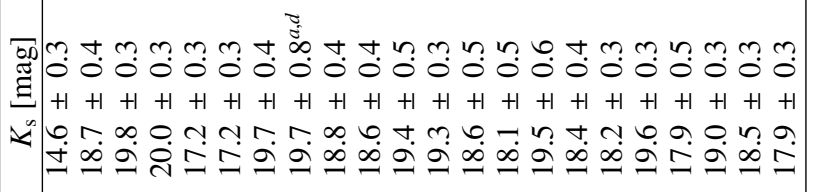 & 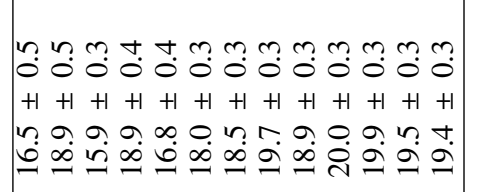 & 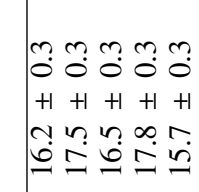 \\
\hline 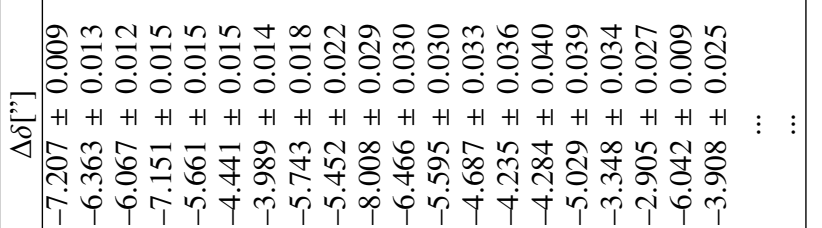 & 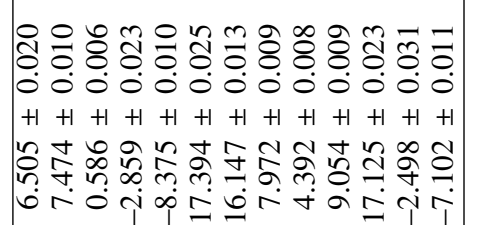 & $\begin{array}{lll}\vdots & \vdots & \vdots\end{array}$ \\
\hline 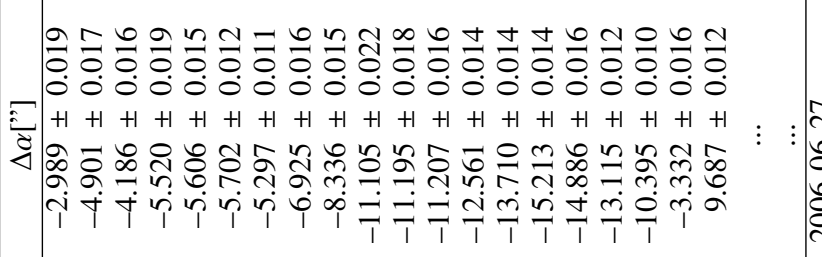 & 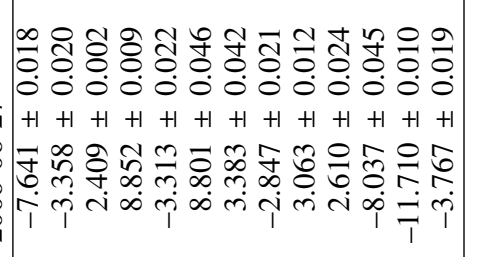 & $\vdots \quad \vdots$ \\
\hline 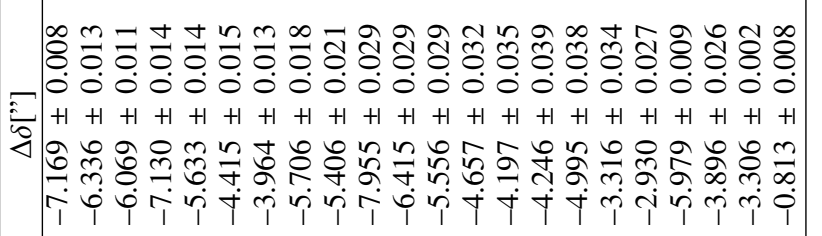 & 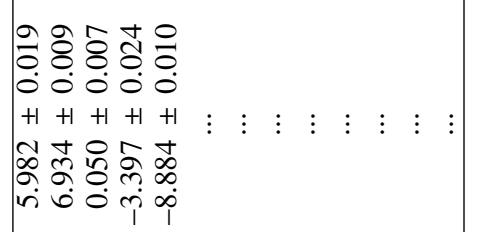 & 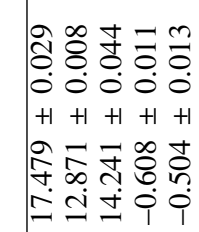 \\
\hline 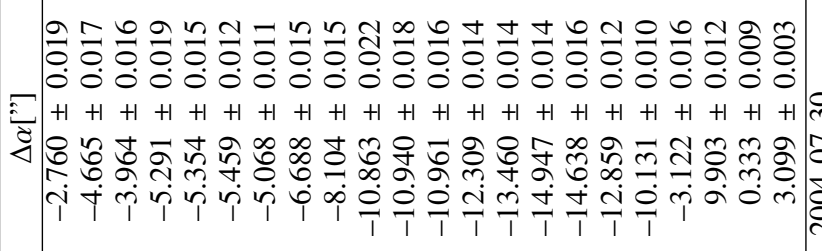 & 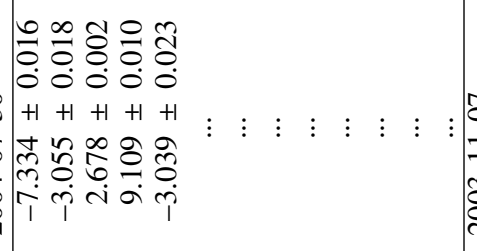 & 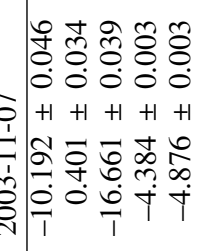 \\
\hline 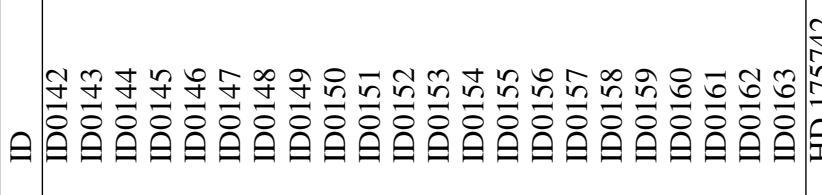 & 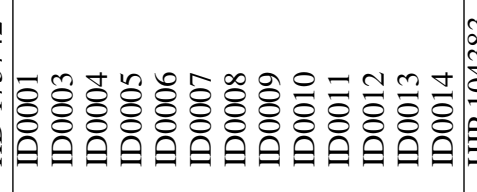 & $\begin{array}{l}0 \\
0 \\
0\end{array}$ \\
\hline
\end{tabular}


M. Ammler-von Eiff et al.: Multiplicity of UMa group members

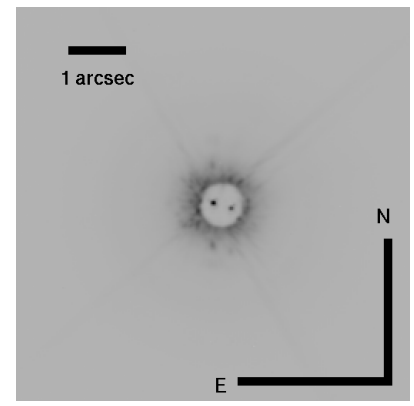

Fig. D.1. The visual binary HIP 104383 A below the semi-transparent coronagraphic mask. The binary has already been characterised (Balega et al. 2004). The full frame exposing companion candidates is shown in Fig. C.4. The magnitudes of the binary components differ by 0.45 mag in the $K_{\mathrm{s}}$ band.

\section{Appendix E: Detection limits}
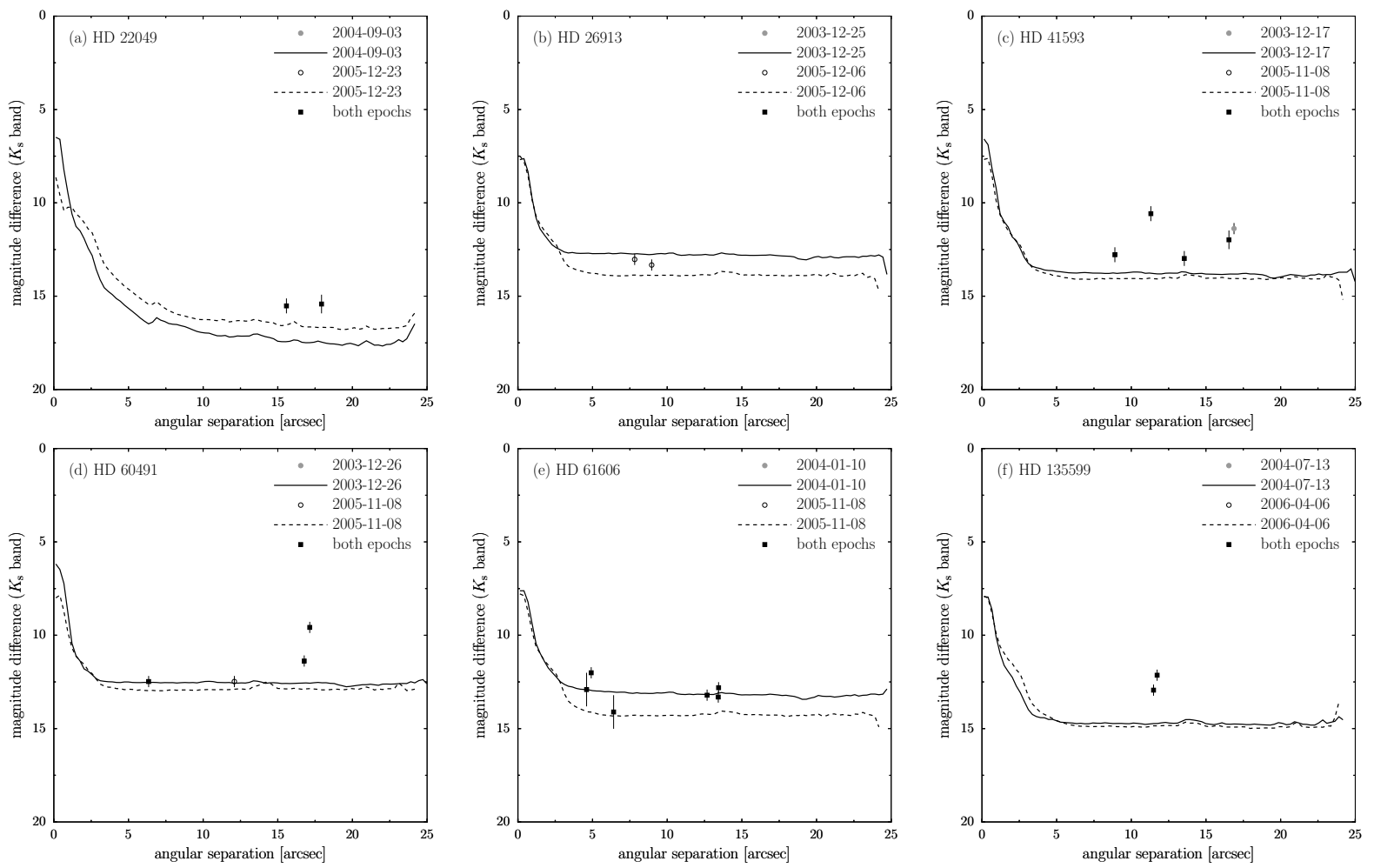

Fig. E.1. Dynamic range curves are shown for stars with candidates and observations in two epochs: HD 22049, HD 26913, HD 41593, HD 60491, HD 61606, and HD 135599. The lines show the $10 \sigma$ detection limits for the 1 st and 2 nd epoch as is indicated in the legend. The adoption of a $5 \sigma$ limit to describe the visual detection of candidates would lower the curves by $2.5 \log 2=0.75$ mag. Furthermore, the curves can vary by $0.3 \mathrm{mag}$ due to the uncertainty of the transmission of the coronagraph. Filled circles indicate candidates found in the $1 \mathrm{st}$ epoch, open circles those in the 2 nd epoch, and filled squares those seen in both epochs. 
A\&A 591, A84 (2016)
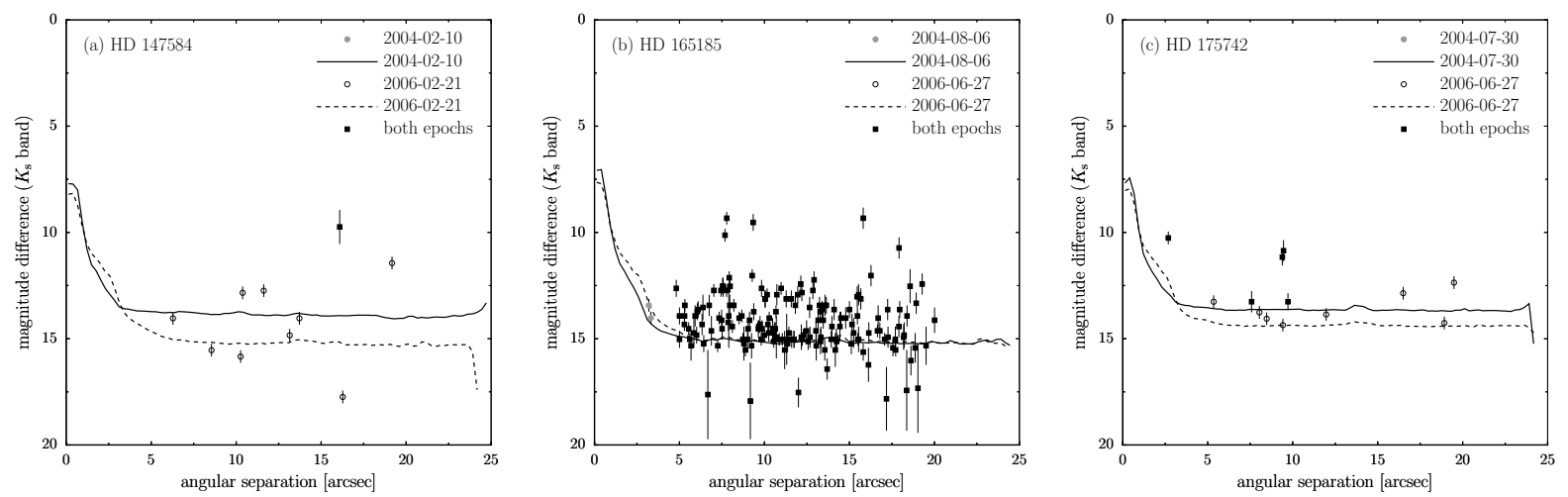

Fig. E.2. Similar to Fig. E.1 for HD 147584, HD 165185, and HD 175742. Some data points are below the detection limits owing to unreliable magnitude measurements (discussed in the text).
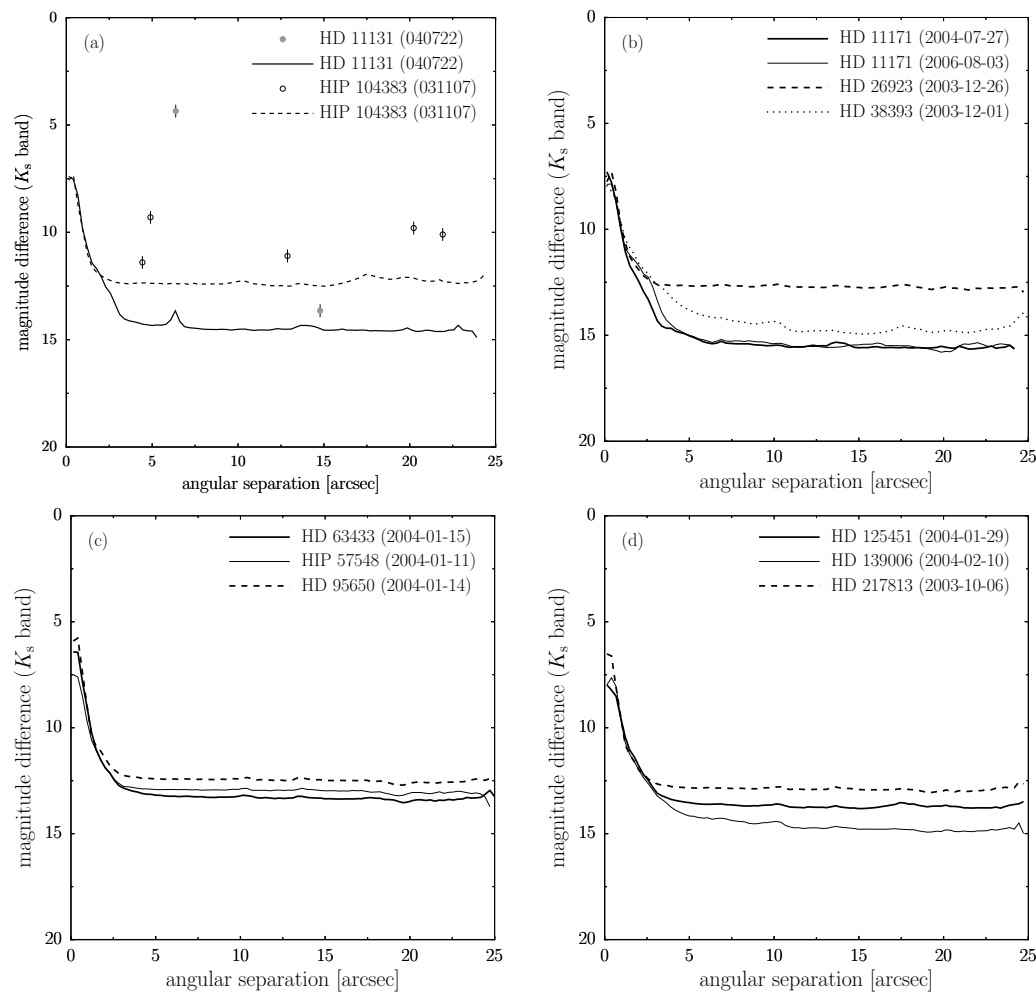

Fig. E.3. Dynamic range curves are shown for stars with candidates detected in a single epoch (HD 11131 and HIP 104383) and for all the other stars without any candidates. 
M. Ammler-von Eiff et al.: Multiplicity of UMa group members
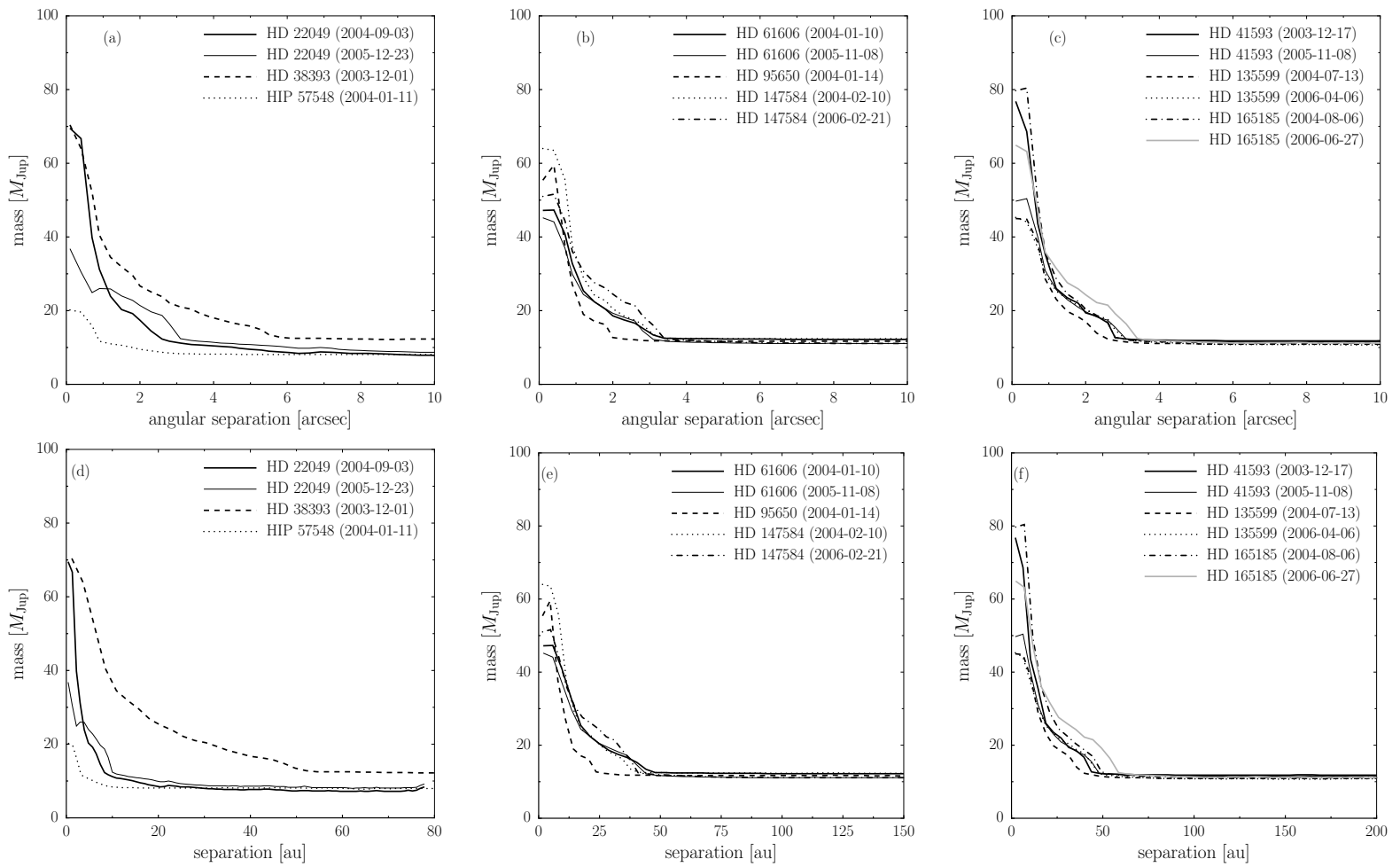

Fig. E.4. Top row: detection limits on mass as a function of angular separation. Bottom row: the same as a function of separation in linear scale. Based on the dynamic range curves presented in Figs. 2 and E.1-E.3 and on evolutionary models (DUSTY00 and COND03) assuming an age of 500 Myr. The layout follows Figs. E.3b-d.
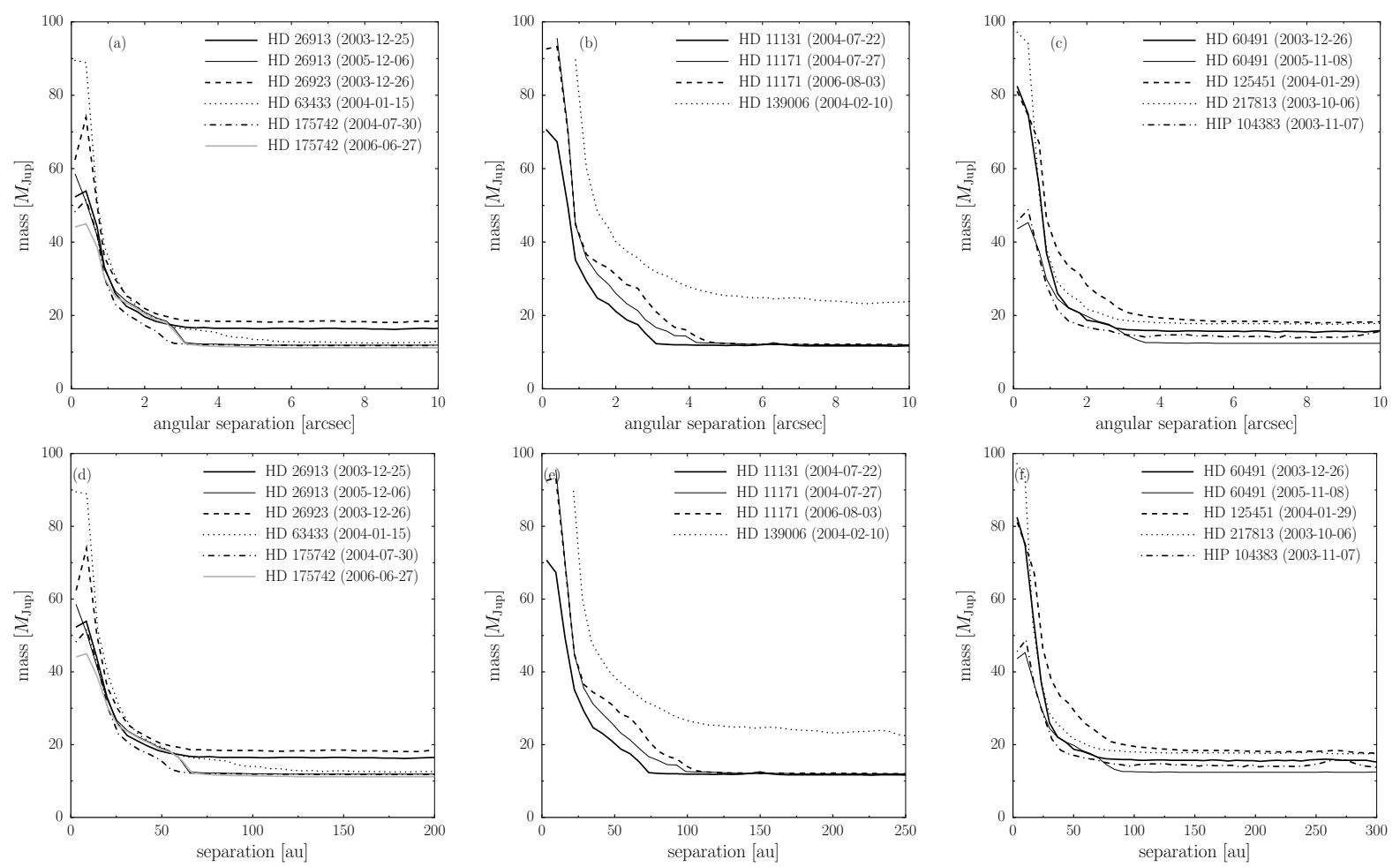

Fig. E.5. Same as in Fig. E.4 for the remaining objects. 
Table E.1. Detection limits derived from exposures of different epochs.

\begin{tabular}{|c|c|c|c|c|c|c|c|c|c|c|c|}
\hline \multirow[t]{3}{*}{ Star } & \multirow[t]{3}{*}{ Epoch } & \multirow{3}{*}{$\begin{array}{c}\text { Exp. time } \\
\text { on target } \\
\text { [s] }\end{array}$} & \multicolumn{9}{|c|}{ Min. separation $[\mathrm{au}]$} \\
\hline & & & & $12 M_{\text {Jup }}$ & & & $20 M_{\text {Jup }}$ & & & $35 M_{\text {Jup }}$ & \\
\hline & & & $100 \mathrm{Myr}$ & $500 \mathrm{Myr}$ & $1 \mathrm{Gyr}$ & $100 \mathrm{Myr}$ & $500 \mathrm{Myr}$ & $1 \mathrm{Gyr}$ & $100 \mathrm{Myr}$ & $500 \mathrm{Myr}$ & $1 \mathrm{Gyr}$ \\
\hline HD 11131 & $2004-07-22$ & 720 & 20.8 & 86.7 & $\cdots$ & 17.6 & 50.7 & 96.7 & $<3.2$ & 22.3 & 34.8 \\
\hline HD 11171 & 2004-07-27 & 495 & 27.0 & 182.1 & $\ldots$ & 22.5 & 63.9 & 220.8 & 14.8 & 29.3 & 50.3 \\
\hline HD 11171 & $2006-08-03$ & 1680 & 27.7 & 247.7 & $\ldots$ & 22.5 & 75.5 & 255.5 & 14.5 & 32.9 & 66.2 \\
\hline HD $22049^{a}$ & 2004-09-03 & 2475 & 2.4 & 8.7 & 18.4 & 1.9 & 5.0 & 8.8 & $<0.4$ & 2.6 & 3.8 \\
\hline HD 22049 & $2005-12-23$ & 720 & $<0.4$ & 10.7 & 29.1 & $<0.4$ & 7.2 & 10.8 & $<0.4$ & 0.7 & 4.4 \\
\hline HD 26913 & $2003-12-25$ & 864 & 17.1 & 515.8 & $\ldots$ & 13.8 & 40.4 & 516.3 & $<2.8$ & 18.6 & 27.2 \\
\hline HD 26913 & $2005-12-06$ & 630 & 16.4 & 97.0 & $\ldots$ & 12.3 & 45.5 & 112.0 & $<2.8$ & 18.0 & 29.1 \\
\hline HD 26923 & $2003-12-26$ & 630 & 19.0 & $\ldots$ & $\ldots$ & 16.0 & 52.1 & $\ldots$ & $<2.9$ & 20.7 & 33.2 \\
\hline HD $38393^{b}$ & $2003-12-01$ & 1673 & 9.6 & 96.4 & $\ldots$ & 7.5 & 31.1 & 97.8 & $<1.2$ & 10.6 & 21.0 \\
\hline $\mathrm{HD} 41593^{c}$ & $2003-12-17$ & 1260 & 13.3 & 60.9 & $\ldots$ & 10.2 & 30.2 & 65.5 & 3.1 & 14.7 & 20.7 \\
\hline HD 41593 & $2005-11-08$ & 630 & 11.4 & 53.8 & $\ldots$ & 8.3 & 30.1 & 56.2 & $<2.1$ & 12.6 & 20.0 \\
\hline HD $60491^{d}$ & $2003-12-26$ & 1855 & 23.0 & $\ldots$ & $\ldots$ & 19.7 & 45.7 & $\ldots$ & 10.1 & 24.5 & 32.0 \\
\hline HD 60491 & 2005-11-08 & 560 & 16.5 & $\ldots$ & $\ldots$ & 10.5 & 48.8 & $\ldots$ & $<3.3$ & 18.8 & 29.7 \\
\hline HD 61606 & 2004-01-10 & 560 & 11.0 & 345.3 & $\ldots$ & 7.2 & 25.6 & 344.3 & $<1.9$ & 12.2 & 18.0 \\
\hline HD 61606 & $2005-11-08$ & 630 & 9.1 & 45.6 & $\cdots$ & 4.1 & 26.4 & 46.3 & $<1.9$ & 10.5 & 16.9 \\
\hline HD 63433 & $2004-01-15$ & 980 & 21.4 & $\ldots$ & $\ldots$ & 17.6 & 46.5 & $\ldots$ & 11.1 & 23.2 & 33.2 \\
\hline HIP 57548 & 2004-01-11 & 630 & $<0.5$ & 2.9 & 8.2 & $<0.5$ & 0.7 & 3.0 & $<0.5$ & $<0.5$ & $<0.5$ \\
\hline HD 95650 & 2004-01-14 & 630 & 7.9 & 30.9 & $\ldots$ & 6.4 & 13.7 & 32.3 & $<1.6$ & 8.6 & 11.8 \\
\hline HD 125451 & 2004-01-29 & 630 & 31.7 & $\ldots$ & $\ldots$ & 25.7 & 91.0 & $\ldots$ & 10.4 & 35.8 & 63.7 \\
\hline HD $135599^{e}$ & 2004-07-13 & 1080 & 10.8 & 43.0 & $\ldots$ & 6.4 & 22.8 & 43.7 & $<2.1$ & 11.9 & 17.5 \\
\hline HD 135599 & 2006-04-06 & 1680 & 10.0 & 49.8 & $\ldots$ & 4.6 & 32.2 & 50.5 & $<2.1$ & 11.6 & 19.7 \\
\hline HD 139006 & $2004-02-10$ & 620 & 52.2 & $\ldots$ & $\ldots$ & 39.3 & $\ldots$ & $\ldots$ & 24.7 & 60.4 & 140.0 \\
\hline HD 147584 & 2004-02-10 & 665 & 11.4 & $\ldots$ & $\ldots$ & 9.7 & 25.3 & $\ldots$ & $<1.6$ & 12.4 & 17.5 \\
\hline HD 147584 & 2006-02-21 & 630 & 10.7 & 47.4 & 292.1 & 8.1 & 32.7 & 49.3 & $<1.6$ & 12.0 & 23.7 \\
\hline HD $165185^{f}$ & 2004-08-06 & 810 & 15.5 & 54.6 & $\ldots$ & 13.0 & 36.2 & 56.1 & 7.8 & 16.8 & 25.3 \\
\hline HD 165185 & 2006-06-27 & 1680 & 15.4 & 65.5 & $\ldots$ & 12.5 & 47.4 & 67.2 & $<2.3$ & 17.2 & 33.6 \\
\hline HD 175742 & $2004-07-30$ & 540 & 16.4 & 77.5 & $\ldots$ & 12.9 & 33.1 & 88.9 & $<2.9$ & 17.7 & 24.4 \\
\hline HD 175742 & 2006-06-27 & 1680 & 15.1 & 70.0 & $\ldots$ & 9.0 & 44.8 & 71.1 & $<2.9$ & 16.8 & 26.8 \\
\hline HD $217813^{g}$ & $2003-10-06$ & 1146 & 23.3 & $\ldots$ & $\ldots$ & 19.5 & 60.1 & $\ldots$ & 12.9 & 25.0 & 39.3 \\
\hline HIP 104383 & 2003-11-07 & 630 & 16.5 & $\ldots$ & $\ldots$ & 12.5 & 35.5 & $\ldots$ & $<3.6$ & 18.5 & 28.3 \\
\hline
\end{tabular}

Notes. (1) Name of the target star. - (2) Epoch of exposure (average if several visits have been co-added, see below). - (3) On-target exposure time (total exposure time on target if several visits have been co-added). - (4-12) lower limit on the separation of detectable objects for masses of 12, 20, and $35 M_{\mathrm{Jup}}$ and ages of $100 \mathrm{Myr}, 500 \mathrm{Myr}$, and $1 \mathrm{Gyr}$, respectively. The relation of masses and magnitudes has been interpolated in the DUSTY00 and COND03 grids. Several targets have been observed repeatedly: ${ }^{(a)}$ 2, 3, and 5 Sep. 2004; ${ }^{(b)} 4$ and 22 Nov. 2003 and 4 Jan. 2004 ; (c) 22 Nov. 2003 and 11 Jan. 2004; ${ }^{(d)} 22$ Nov. 2003, 10 and 15 Jan. 2004; ${ }^{(e)} 4$ and 22 Jul. 2004; ${ }^{(f)} 4$ Jul. and 9 Sep. $2004 ;^{(g)} 6$ and 7 Oct. 2003.

Table E.2. Apparent motion in right ascension and declination predicted for all stars with at least one candidate observed in two epochs.

\begin{tabular}{lllrr}
\hline \hline Star & $\begin{array}{l}\text { 1st epoch date } \\
\text { yyyy mm dd }\end{array}$ & $\begin{array}{l}\text { 2nd epoch date } \\
\text { yyyy mm dd }\end{array}$ & $\Delta \alpha \cos \delta[\mathrm{mas}]$ & $\Delta \delta[\mathrm{mas}]$ \\
\hline HD 22049 & $2004-09-03$ & $2005-12-23$ & $-1734.94 \pm 0.36$ & $-155.21 \pm 0.28$ \\
HD 41593 & $2003-12-17$ & $2005-11-08$ & $-190.06 \pm 1.40$ & $-193.41 \pm 0.81$ \\
HD 60491 & $2003-12-26$ & $2005-11-08$ & $-128.10 \pm 2.43$ & $-72.76 \pm 1.25$ \\
HD 61606 & $2004-01-10$ & $2005-11-08$ & $185.25 \pm 1.40$ & $-499.95 \pm 0.57$ \\
HD 135599 & $2004-07-13$ & $2006-04-06$ & $398.45 \pm 1.52$ & $-255.43 \pm 1.09$ \\
HD 147584 & $2004-02-10$ & $2006-02-21$ & $410.33 \pm 0.51$ & $213.21 \pm 0.88$ \\
HD 165185 & $2004-08-06$ & $2006-06-27$ & $234.38 \pm 1.17$ & $12.16 \pm 0.60$ \\
HD 175742 & $2004-07-30$ & $2006-06-27$ & $276.77 \pm 1.05$ & $-537.13 \pm 1.20$ \\
\hline
\end{tabular}

Notes. Based on proper motion and stellar parallactic motion measured by HIPPARCos (Tables A.1 and A.2). These numbers are used to identify non-moving background stars next to the targets. 